\title{
Domains in a Realizability Framework ${ }^{1}$
}

\author{
Roberto M. Amadio ${ }^{2}$ \\ Labo. Informatique, Ecole Normale Supérieure, \\ 45 rue d'Ulm, F-75230 Paris. (amadio@dmi.ens.fr)
}

\begin{abstract}
We aim to relate and develop various approaches to a theory of domains in a realizability framework.

In the first part we develop the theory in an arbitrary partial cartesian closed category and, as a particular instance, in the category of partial equivalence relations (per) over an arbitrary partial combinatory algebra (pca). In this way one obtains the category of separated pers that may be regarded as a universe of partially ordered sets and monotone maps with strong closure properties.

Pursuing the analogy with domain theory one tries to build a category of directed complete partial orders and continuous maps. We consider two main approaches: one based on Kleene's pca of numbers and partial recursive functions and the other based on $S$ cott's $D_{\infty} \lambda$-model.

Contents: 0. Introduction, 1. Dominance and Intrinsic Preorder, 2. Separated Partial Equivalence Relations (Eper), 3. N-completeness, 4. E-connection and Extensionality, 5. D-completeness and Uniformity, 6. Conclusion.
\end{abstract}

\section{Introduction}

The general and vague problem is to develop a theory in which data types can be regarded as sets. A number of examples show that classical set theory is not well-suited to this purpose. However it has become more and more clear that this program can be achieved if one takes a constructive attitude. In particular we will be interested in settheoretic structures based on realizability.

A model for a programming language should include some notion of effectivity and/or computability. In the kind of models we are going to present the underlying realizability structure plays the role of an abstract machine. A data is represented as a collection of extensionally equivalent implementations. A map from data $A$ to data $B$ is a function that can be actually implemented by a realizer and that transforms equivalent implementations of a data in A into equivalent implementations of a data in B. This aspect of the model is relevant for the interpretation of subtyping.

The fact that all operations have to be realized leads to very interesting properties of the model reflecting its computational nature. A typical example is the validity of a "Uniformity Principle" which plays an important role in the interpretation of second order quantification as intersection.

There have been several attempts in this direction. It seems fair to say that this program was first pushed by Dana Scott and his students: McCarty [84], and Rosolini[86], whose work relates in particular to the effective topos (Hyland[82], but see also Mulry[81] for another approach). Recently there has been another wave of results in Amadio[89], Abadi\&Plotkin[90], Freyd\&al.[90], Phoa[90]. We wish to analyse them from two points of view:

(1) Success in combining interesting properties of domain-theoretic and realizability models. For example, for the former: solution of recursive domain equations (Scottl72], Wand[80], Smyth\&Plotkin[82]), existence of a universal (homogeneous) domain (Scott[76], Gunter\&Jung[90], Droste\&Göbel[90]), abstract account of effective computations (Smyth[77]); and for

\footnotetext{
${ }^{1}$ A preliminary version of this paper appears as LIENS-TR 19-90.

2 Work done while on leave from: Dipartimento di Informatica, Università di Pisa. Research partly supported by the Joint Collaboration Contract "Typed Lambda Calculus" of the EEC and by the Basic Research Action "Categorical Logic in Computer Science" of the EEC.
} 
the latter: simple interpretations of polymorphism (Hyland[89], Carboni\&al.[88], Longo\&Moggi[88]) and subtyping (Bruce \&Longol90]).

(2) Conceptual economy of the solution, that is trying to have a theory that is at once general in that it allows to explain and relate different approaches and simple in that it makes a careful analysis of the axioms introduced.

\subsection{External vs. Internal Logic}

We have to point-out that the results of such analysis may depend on the tools we use. We will try to work as much as we can with a generic partial combinatory algebra $D$ (henceforth pca, definition in 2.1.1) and in full (reflective) subcategories of the category of partial equivalence relations over $D$ (henceforth per $_{D}$ ).

We will not be able to carry on all the program at this level of generality, however, in view of new developments, we think that it is important to stress which results are general and which results depend on the specific pers and pcas we are dealing with.

A subtle point is the choice between an external classical logic (say a system like ZF) and the internal logic of a realizability universe. Living in a set-theoretic realizability framework it is clear after a few examples that usual definitions (e.g. that of $\mathrm{N}$ completeness, see 3.1) do not lead to the expected theorems unless they are given a constructive reading.

In this sense one receives benefits by thinking at the constructive interpretation of logical operators. This approach can be made rigorous by using the internal logic of the effective topos, basically a higher order intuitionistic type theory that includes principles like the countable axiom of choice $\left(\mathrm{AC}_{\omega}\right)$, the computability of all the maps on natural numbers (Church Thesis), the Uniformity Principle and Markov Principle (see Hyland[82]). Reasoning in this non-standard logic is prone to errors induced by our habit of working in classical logic. Moreover there is an obvious problem of communication with the overwhelming majority of the mathematical community.

The moral that we draw from this situation is that when developing the theory one should make a sensible use of the internal logic. In particular one should try to understand what is the effect of a constructive interpretation of a definition on the structure of the mathematical object we are dealing with. This may involve some tiresome computation on realizers, but we will be rewarded by a stronger intuition of "what is going on" that may lead to new insights. Every logical statement that we formulate in the following part of the paper should be read classically as a sentence of, say, ZF set theory.

\subsection{Overview}

The paper is supposed to be a gentle and systematic introduction, requiring a minimum of logical and categorical apparatus, to a series of concepts that were mostly introduced or suggested by other people. However, to the best of our knowledge, this is the first place where a systematic analysis of the various approaches mentioned above and an investigation of their relationships is started. In a sense we feel that this is our main contribution or, at least, the one that took the greatest effort. ${ }^{3}$

In $\$ 1$ we develop a notion of intrinsic pre-order working in an arbitrary partial cartesian closed category (pccc). Every pccc has an object $\Sigma$ that classifies the admissible monos and that can be regarded as an abstraction of Sierpinski space. The following isomorphism holds: $a \rightarrow \Sigma \cong a \rightarrow 14$; hence given $f: a \rightarrow \Sigma$ and $x: 1 \rightarrow a$ we have an induced notion of convergence for $f x$. Given two points of an object $a$, say $x, y: 1 \rightarrow a, x$ is intrinsically less than $y$ if any map $f: a \rightarrow \Sigma$ that converges on $x$ also converges on $y$. Maps

3 In this process of revision and generalization a number of conceptual simplifications have been made.

$4 \mathrm{a} \rightarrow 1$ are the "partial" maps from a into the terminal object. 
automatically preserve the intrinsic pre-order. A separated object is an object in which the intrinsic pre-order is a partial order (i.e. anti-symmetry holds).

In $\$ 2$ we restrict our attention to a pccc of pers over an arbitrary pca. The convergence predicate of the pca naturally induces on any given per a family of semi-computable predicates, that, on the other hand, gives rise to a family of admissible monos generating the pccc. Separated pers (Iper) are those pers whose points are separated by the topology induced by the semi-computable subsets. They are shown to be a full reflective sub-category of per and therefore they enjoy all the completeness properties of the larger category. The category of separated pers can be seen as a universe of partially ordered sets and monotone maps.

In $\$ 3$ the notion of $N$-completeness is introduced. $\Sigma$ per contains a natural number object $(\mathrm{N})$. Given a $\Sigma$ per A we will say that A is N-complete if every chain (w.r.t. the intrinsic order) described by a map from $\mathrm{N}$ to $\mathrm{A}$ has a supremum in $\mathrm{A}$. When working on Kleene's pca $(\omega, \cdot)$ it will turn out that all realized maps between $N$-complete $\Sigma$ per $\left(c_{N} \Sigma\right.$ per $)$ are continuous in the sense of preserving suprema of such chains.

In $\$ 4$ the notion of $\Sigma$-connected object (or equivalently $\Sigma$-linked object) is presented. Its motivation arises from the need of giving a sufficient condition for the coincidence of intrinsic and pointwise orders on products. On the other hand the problem of having strong completeness properties for $c_{N} \Sigma$ per ${ }_{\omega}$ leads to the introduction of extensional pers (exper). The notion of extensionality implies those of separation and $\Sigma$-connection. By definition every extensional per A can be regarded as a sub-poset of a "functional" per $B \rightarrow \Sigma$. This assumption has two important consequences: (a) it is possible to exhibit an operator that, uniformly, given any (N-)ascending chain computes its lub; (b) since $B \rightarrow \Sigma$ is $N$ complete it is possible to extend every extensional per to an $\mathrm{N}$-complete extensional per hence showing the reflection of $c_{N}$ exper ${ }_{\omega}$ into exper $\omega^{*}$

In $\$ 5$ we take the point of view that the notion of computation should be modelled at a suitable level of abstraction, say in terms of ordered sets and continuous transformations, rather than at the level of indices of Turing machines. Working over a pca $D_{\text {dcpo }}$ with the structure of a directed complete partial order we discuss the notion of D-complete $\Sigma$ per ( $c_{D} \Sigma$ per). A per is D-complete if, when regarded as a relation, it is closed under directed sets w.r.t. the D-order. The category of $\mathrm{c}_{\mathrm{D}}(\Sigma)$ per ${ }_{\mathrm{Ddcpo}}$ is reflective in $(\Sigma)$ per $_{\mathrm{Ddcpo}}$ and its pointed objects interpret a least fixed point combinator. Next we consider a pca $D_{\infty}$ built via the inverse limit technique and we impose a condition of uniformity on the $c_{D} \Sigma$ pers $\left(c_{D} u \Sigma\right.$ per). Under this condition ${ }_{C_{D}} u \Sigma$ per ${ }_{D_{\infty}}$ turns out to be a complete ultra-metric space. Thanks to the contractiveness of the first-order type constructors it is possible to solve domain equations up-to-equality. Moreover if we impose that $D_{\infty}$ is in $\operatorname{sfp}^{5}, \mathrm{D}_{\mathrm{sfp}}$, we can look at the full subcategory $\mathrm{c}_{\mathrm{D}} \mathrm{u} \Sigma$ per $_{\mathrm{D}_{\text {sfp }}}$ as at sfp domains and continuous maps (w.r.t. the intrinsic order). The crucial theorem shows that the quotient space is a cpo. Its proof relies on techniques apparently unrelated to those employed in $\$ 3$.

The following diagram summarizes the relationships among the main categories discussed above:

$$
\begin{aligned}
& c_{N} \operatorname{exper} \omega C_{>} \text {exper } \\
& c_{N} \Sigma \text { per } \omega \subset \Sigma \text { per }_{D} \subset_{>} \text {per }_{D} \\
& \hat{v} \\
& c_{D} u \Sigma \text { per }_{\text {Dsfp }} \subset c_{D} \Sigma \text { per }
\end{aligned}
$$

where: ${ }^{6} \quad c \equiv$ full subcategory $\quad c_{>} \equiv$ full reflective subcategory.

\footnotetext{
5 sfp stands for Sequences of Finite inductive Partial orders. The category of sfp domains and continuous maps was introduced by G. Plotkin. sfp domains are also referred to with the name of bifinite domains.

6 For the reader's convenience we recall: $D \equiv$ pca. $\omega \equiv$ Kleene's pca. Ddcpo $\equiv a$ specific pca in dcpo. Dsfp $\equiv a$ specific pca in sfp. per $\equiv$ pers. $\Sigma \equiv$ separated. ex $\equiv$ extensional. $\mathrm{CN}_{\mathrm{N}} \equiv \mathrm{N}$-completeness. $\mathrm{CD}_{\mathrm{D}} \equiv \mathrm{D}$-completeness.
} 


\section{Dominance and Intrinsic Preorder}

In this section we introduce some basic notions about partial cartesian closed categories (pccc) and their properties.

Every pccc has an object $\Sigma$, called dominance, that classifies the admissible subobjects in the same sense as the object of truth-values $\Omega$ classifies arbitrary subobjects in a topos.

The object $\Sigma$ induces a preorder $\leq_{\mathrm{a}}$ on the points of every object a (i.e. maps from the terminal object into a). Following Rosolini[86] we focus on the full subcategory of separated objects, that is composed of those objects for which $\leq_{\mathrm{a}}$ is antisymmetric.

We collect some basic properties of this subcategory that will be extensively used in the following sections.

\subsection{Partial Cartesian Closed Categories (pccc) 7}

It is standard to consider an equivalence class of monos on an object as a generalized notion of subset. A partial map from a to $b$ can then be represented as a total map from a subset of $a$ to $b$. In many interesting examples the domain of convergence of a partial map is not arbitrary. E.g. it is open, recursively enumerable, etcetera. It is then reasonable to look for a corresponding categorical notion of admissible mono as specified by the following definition.

\subsubsection{Definition (Admissible family of monos) ${ }^{8}$}

An admissible family of monos $\mathrm{M}$ for a category $\mathrm{C}$ is a collection $(\mathrm{M}(\mathrm{a}) / \mathrm{a} \in \mathrm{Ob} \mathrm{C}$ ) s.t.:

(1) If $m \in M(a)$ then $m$ is a mono $m: d \rightarrow a$.

(2) The identity on $a$ is in $M(a): i d_{a} \in M(a)$.

(3) $\mathrm{M}$ is closed under composition i.e.

if $m_{1}: a \nrightarrow b \in M(b)$ and $m_{2}: b \rightarrow c \in M(c)$ then $m_{2} m_{1}: a \rightarrow c \in M(c)$.

(4) $M$ is closed under pullbacks i.e.

if $m: d \rightarrow b \in M(b)$ and $f: a \rightarrow b$ then $f^{-1}(m) \in M(a)$.

\subsubsection{Fact (the related category of partial maps)}

An admissible family of monos $M$ on $C$ enjoys properties sufficient for the construction of a related category of partial maps $\mathrm{pC}$. A representative for a partial map from $\mathrm{a}$ to $\mathrm{b}$ is a pair of maps in $C,(m, f)$, such that $m: d \rightarrow a \in M(a)$ determines the domain and $f: d \rightarrow b$ the functional behavior. The category $\mathrm{pC}$ has the same objects as $\mathrm{C}$ and as morphisms equivalence classes of representatives of partial maps, namely:

$p C[a, b] \triangleq\{[m, f] / m: d \rightarrow a \in M(a), f: d \rightarrow b\}$

where $(m, f)$ is equivalent to $\left(m^{\prime}, f^{\prime}\right)$ iff they have the same domain and codomain, and there is a map that makes $m$ isomorphic to $m^{\prime}$ in the slice category $C / a$, and $f$ isomorphic to $f^{\prime}$ in the slice category $\mathrm{C} / \mathrm{b}$. To specify domain and codomain of a (representative) for a partial map we write $[m, f]: a \rightarrow b((m, f): a \rightarrow b)$.

\subsubsection{Definition $(P c c c)$}

Let $M$ be an admissible collection of monos on the category $C .(C, M$ ) is a pccc (partial cartesian closed category) iff $\mathrm{C}$ is cartesian and for any $\mathrm{b}$ there is a a functor partial exponent on $\mathrm{b}$ pexp $\mathrm{p}_{\mathrm{b}} \mathrm{pC} \rightarrow \mathrm{C}$ that is right adjoint to the product functor $\_\mathrm{b}: \mathrm{C} \rightarrow \mathrm{pC}$ that is: $\mathrm{pC}[\times \mathrm{b}, \ldots] \cong \mathrm{C}\left[\right.$, pexp $\_$.

$\mathrm{u} \equiv$ uniformity. This is just a mnemonic schema. Note that we are dealing with different pcas. In general the pca of the contained category is an instance of the pca of the containing category. Things make sense only after instantiation of the latter (containing) with the former (contained).

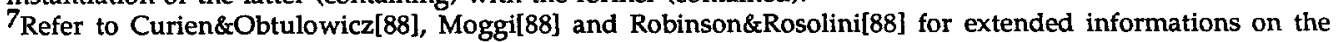
origins and the development of the theory. Longo\&Moggi[84] is the first place were we found definition 1.1.3.

8 We often abbreviate functional composition $\mathrm{f} g$ with $\mathrm{fg}$. 
By instantiating the natural isomorphism one obtains the following version of "currying": $a \times b \rightarrow c \equiv a \rightarrow(b \rightarrow c)$, where we write more suggestively $b \rightarrow c$ for pexp $(c)$. Indeed by virtue of the previous isomorphism we can safely confuse $b \rightarrow c$ with $p C[b, c]$. Finally the standard notion of lifting can be defined as $(a)_{\perp} \triangleq 1 \rightarrow$ a.

\subsubsection{Fact (Dominance)}

In every pccc there is an object $\Sigma \triangleq(1)_{\perp} \triangleq 1 \rightarrow 1$, called dominance, that classifies the monos in $M: M() \cong C[, \Sigma]: C^{o p} \rightarrow$ Set. The following isomorphism holds: $a \rightarrow \Sigma \cong a \rightarrow 1$.

\subsection{Intrinsic Pre-order}

In a pccc the maps from an object a to the dominance $\Sigma$ play the role of convergence tests. Such tests induce a pre-order on the points of an object. The idea of ordering points by tests bears a striking analogy with the one encountered in, so-called, operational semantics of ordering terms by observations. However a crucial difference is that in our case the preorder does not depend on the language at hand but only on a global category on which one can interpret a variety of constructs.

\subsubsection{Convention (Points and convergence predicate)}

We write $x: a$ to indicate that $x$ is a point of $a$, that is a morphism $x: 1 \rightarrow a$. Since we will be dealing with (p)ccc we confuse points in the objects $a \rightarrow b$ and $a \rightarrow b$ with morphisms, respectively, in $C[a, b]$ and $p C[a, b]$. E.g. $f: a \rightarrow b$ can be seen both as a morphism from $a$ to $b$ and as a point in $a \rightarrow b$.

We introduce a convergence predicate, $\downarrow$, as follows: if $x: a, p \equiv[m, f]: a \rightarrow b$, with $m: d r a$, $\mathrm{f}: \mathrm{d} \rightarrow \mathrm{b}$ then we write: $\mathrm{px} \downarrow \Leftrightarrow \exists \mathrm{h}: 1 \rightarrow \mathrm{d}$. $(\mathrm{mh}=\mathrm{x})$. By virtue of the isomorphisms in 1.1.4 we write both $p: a \rightarrow b$ and $p: a \rightarrow(b)_{1}$.

\subsubsection{Definition (Intrinsic preorder) ${ }^{9}$}

Let $(C, M)$ be a pccc and a an object. Define a preorder $\leq \mathrm{a}$ on the points of a as:

if $x, y: a$ then $x \leq a y$ iff $\forall p: a \rightarrow \Sigma$. $(p x \downarrow \supset p y \downarrow)$.

The intuition is that $x$ is less then $y$ in a if every convergence test p:a $\rightarrow \Sigma$ that succeeds on $x$ also succeeds on $y$. We also write $p x \leqslant$ py for px $\downarrow \supset p y \downarrow$, and px py for px $\downarrow \Leftrightarrow p y \downarrow$.

\subsubsection{Definition (category of $\Sigma$-objects)}

Given a pccc $(C, M)$ with dominance $\Sigma$ we denote with $\Sigma C$ the full subcategory of $C$ whose objects enjoy the property that the intrinsic preorder is anti-symmetric. An object a such that $\leq_{\mathrm{a}}$ is a partial order is called $\Sigma$-object or, equivalently, separated object.

\subsubsection{Proposition (Basic properties)}

Let $(C, M)$ be a pccc with dominance $\Sigma$. Then:

(1) Maps preserve the intrinsic preorder.

(2) $\Sigma$-objects are closed under subobjects.

(3) Moreover if $(C, M)$ has enough points and a is an object then $\Sigma^{a}$ is a $\Sigma$-object. Proof

(1) Let $f: a \rightarrow b$ and $x, y: a$. Suppose $x \leq_{a} y$, then given any $p: b \rightarrow \Sigma$ we have by hypothesis pf $x \leqslant$ pfy, since pf: $a \rightarrow \Sigma$. Hence $f x \leq_{b} f y: b$.

(2) Let $m: a>b$ be $a$ mono and $b$ be $a$-object. If $x$ and $y$ are two distinct points in $a$ then $\mathrm{mx}$ and $\mathrm{my}$ are two distinct points in $\mathrm{b}$. Hence, since $\mathrm{b}$ is a $\Sigma$-object, they are separable by a

$\overline{9}$ Every partial category $\mathrm{pC}$ has a partial ordering on morphisms given by the containment of the convergence spaces. In such partial ordering the total morphisms represent the maximal elements. Therefore the points of an object $a$, i.e. the total maps $x: 1 \rightarrow a$, turn out to be all incomparable. On the other hand the intrinsic preorder may give non-trivial partial orders on the points. 
map $p: b \rightarrow \Sigma$. Then the map pm separates the points $x$ and $y$.

(3) If $f, g: \Sigma^{a}$ and $f \neq g$ then, by the enough point assumption, there is a $x: a$ s.t. $\sim f x \cong g x$. Take $\lambda h: \Sigma^{a} . h x: \Sigma^{a} \rightarrow \Sigma$ as separator for $f$ and $g$.

\subsubsection{Note (on category theoretic notions)}

We recall the notion of full reflective subcategory that will play an important role in the following sections. Suppose that $C$ is a full subcategory of $D$. We say that $C$ is reflective in $D$ if the inclusion functor Incl: $\mathrm{C} \rightarrow \mathrm{D}$ has a left adjoint $\mathrm{L}: \mathrm{D} \rightarrow \mathrm{C}$ (called reflector), hence satisfying: $C\left[L_{\perp}\right] \cong D[$, Incl_]. If $C$ is a full reflective subcategory of $D$ then every functor $S: J \rightarrow C$ having limit in $D$ has (an isomorphic) limit in $C$ (see MacLane[72] for a sketch of the proof). Hence $C$ inherits all the limits and colimits ( $L$ has a right adjoint) of $D$.

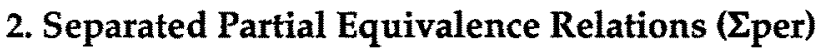

One can build over every partial combinatory algebra (pca) D the category of partial equivalence relations, per $_{D}$, and a family of admissible monos, $M_{D}$. (per ${ }_{D}, M_{D}$ ) turns out to be a pccc with enough points. Besides, per $_{\mathrm{D}}$, the category of separated pers over D, is reflective in perD.

\subsection{Partial Combinatory Algebras and PER}

The category of pers built over a pca has very relevant completeness properties (see Hyland[88], Carboni\&al.[87], Longo\&Moggi[88]). In the following we will take the notion of morphism as fundamental and we will restrict our attention to full, possibly reflective, subcategories.

\subsubsection{Definition (partial combinatory algebra)}

A partial combinatory algebra (pca) is a partial algebraic structure $(D, k, s, \cdot)$ where: $k, s \in D, \cdot: D \times D \rightarrow D$, and $k x y=x, s x y \downarrow, s x y z \cong x z(y z)$.

As usual $\rightarrow$ denotes the partial functional space. $t \downarrow$ denotes the fact that the expression $t$ is defined. $\cong$ is Kleene's equality: $t \cong s$ iff $(t \downarrow \Leftrightarrow s \downarrow) \wedge(t \downarrow \Rightarrow t=s)$. $t=s \Rightarrow t \downarrow \wedge s \downarrow, t s \downarrow \Rightarrow t \downarrow \wedge s \downarrow$.

\section{Proviso}

For the sake of readability we will write programs using $\lambda$-notation. It is intended that terms have to be compiled in the language of combinators according to some translation that simulates conveniently $\lambda$-abstraction.

\subsubsection{Definition (partial equivalence relations)}

Given a pca $D$ the category of pers over $D$ is defined as follows:

Obper $_{D} \triangleq\{A \mid A \subseteq D \times D$ and $A$ is symmetric and transitive $\}$ $\operatorname{per}_{D}[A, B] \triangleq\left\{f: D / A \rightarrow D / B \quad \mid \exists \phi \in D \cdot \forall d \in D \cdot d A d \Rightarrow \phi d \in f\left([d]_{A}\right)\right\}$

where conventionally : $d A e \equiv_{a b r}(d, e) \in A,[d]_{A} \triangleq\{e \in D \mid d A e\}, D / A \triangleq\left\{[d]_{A} \mid d A d\right\}$, $|A| \triangleq\{d \mid d \in D, d A d\}$, if $\phi$ is a realizer for the map $f: A \rightarrow B$ we may denote $f$ with $[\phi]_{A \rightarrow B}$.

\subsubsection{Semi-computable predicates on $D$}

Partiality is explicitly given in D and by generalizing basic facts of recursion theory (i.e. r.e. sets are exactly the domain of computable functions) it also provides a notion of semicomputable predicate on $D$.

(1) $\Sigma(D) \triangleq\{W \mid W \subseteq D, \exists d \in D W=\operatorname{Dom}(d)\}$, where $\operatorname{Dom}(d) \triangleq\{e \mid e \in D, \operatorname{de} \downarrow\}$.

(2) The collection of predicates $\Sigma(D)$ induces a refinement pre-order on $D$ defined as: $\mathrm{d} \leq_{\mathrm{D}} \mathrm{e}$ iff $\forall W \in \Sigma(D) .(\mathrm{d} \in \mathrm{W} \Rightarrow \mathrm{e} \in \mathrm{W})$ 
(3) Observe that the operation of application preserves this pre-order:

$\forall \mathrm{e} \in \mathrm{D} .\left(\mathrm{d} \leq_{\mathrm{D}} \mathrm{d}^{\prime} \Rightarrow\right.$ ed $\left.\leq_{\mathrm{D}} \mathrm{ed}\right)$.

(4) If the pca is not total then $\Sigma(D)$ can be seen as a basis for a topology as:
(a) $\varnothing, D \in \Sigma(D)$.
(b) If $W, W^{\prime} \in \Sigma(D)$ then $W \cap W^{\prime} \in \Sigma(D)$

Hints: (a) Take respectively the always divergent and always convergent map. (b) If $W=\operatorname{Dom}(e)$ and $W^{\prime}=\operatorname{Dom}\left(e^{\prime}\right)$ then $W \cap W^{\prime}=\operatorname{Dom}\left(\lambda d . i f\left(e d \downarrow \wedge e^{\prime} d \downarrow\right)\right.$ then $\left.\downarrow\right)=\operatorname{Dom}\left(\lambda d .(\lambda \times . \lambda y . c)(e d)\left(e^{\prime} d\right)\right)$.

\subsubsection{Examples (pcas)}

(1) An important example of pca is Kleene's $\left(\omega_{,} \cdot\right)$ where $\omega$ are the natural numbers and $n \cdot m$ is the $n$-th Turing machine applied to the input $m$, (given some standard enumeration). In this case: $\Sigma(\omega, \cdot)=R E$, the recursively enumerable sets.

(2) Another canonical example of pca is that of a non-trivial domain $D$ that is a retract ${ }^{10}$ of the partial functional space, written $D \rightarrow D \triangleleft D$, in the category of directed complete partial orders and partial continuous maps (see Plotkin[85]).Then $\Sigma(D)$ is the collection of open sets in Scott's topology.

(3) Examples of pca's in which the collection of points is not separable can be built out of term models. For example if one takes a $\lambda \beta$ term model where the notion of convergence corresponds to reduction to a $\lambda$-abstraction one finds that all terms that are strongly unsolvable are undistinguishable.

\subsection{PER as a Pcce}

We show that given any per, A, $\Sigma(D)$ induces a collection, $\Sigma(A)$, of semi-computable predicates on $A$. From this structure it is easy to obtain a family $M_{D}$ of admissible monos on perD that turns the category into a pccc.

\subsubsection{Definition (Semi-computable subsets of a per)}

Let $A \in$ per $_{D}$, define $\Sigma(A) \triangleq\left\{B \in \operatorname{per}_{D}|D / B \subseteq D / A, \exists W \in \Sigma(D)| A.|\cap W=| B \mid\right\}$.

In other terms $B$ belongs to $\Sigma(A)$ if the equivalence classes in $B$ form a subset of those in $A$ and there is a set $W \in \Sigma(D)$ that separates $B$ from the other equivalence classes in $D / A$. This definition generalizes the notion of semi-computable set in the category of enumerated sets (or equivalently total equivalence relations). In this case an object is a pair $\left(X, e_{X}: \omega \rightarrow X\right)$ where $e_{X}$ is an onto enumeration of $X$ and a subset $Y$ of $X$ is semi-computable iff $e^{-1}(Y)$ is recursively enumerable. Such sets form the basis for what is sometime called Mal'cev topology.

\subsubsection{Closure properties of $\Sigma(\mathbf{A})$}

Assume $D$ non-total pca. Then $\Sigma(A)$ enjoys closure properties analogous to $\Sigma(D)$ (see 2.1.3.(4)): (a) $\varnothing, A \in \Sigma(A)$. (b) If $B_{,} B^{\prime} \in \Sigma(A)$ then $B^{\prime \prime} \in \Sigma(A)$, where $B^{\prime \prime}$ is the per corresponding to the partial partition $D / B \cap D / B '$.

\subsubsection{Definition (Admissible family of monos)}

Define $M_{D}$ as the following family of monos: $m: A^{\prime} \rightarrow A \in M_{D}(A)$ iff $A^{\prime} \in \Sigma(A)$ and $m$ is the inclusion morphism.

Note that the morphism $m$ is realized by the identity (hence $m$ is a coercion in the sense of Bruce\&Longo[88]). It is easy to check that this collection of monos is indeed admissible. The conditions for identity and composition are clear. Let us consider the case for the pullbacks: ${ }^{10}$ We write $D \triangleleft E$ if there is a pair of morphisms $i: D \rightarrow E, j: E \rightarrow D$ such that $j i=i d_{D}$. 
assume $f: A \rightarrow B$ and $m: C \rightarrow B$ with $\phi$ realizer of $f$ and $|B| \cap D o m(\psi)=|C|$. To construct the pullback consider $W^{\prime}=\operatorname{Dom}(\lambda d . \psi(\phi d))$ and the related $C^{\prime}$ admissible subobject of $A$.

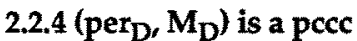

The category (per ${ }_{\mathrm{D}}, \mathrm{M}_{\mathrm{D}}$ ) of pers and partial maps (see 1.1.2) is equivalent to the category pper $_{D}$ defined as follows:

$$
\begin{aligned}
& O b_{\text {pperD }} \triangleq \mathrm{Ob}_{\text {perD }} \\
& \operatorname{pper}_{D}[A, B] \triangleq \\
& \triangleq\left\{\mathrm{f}: \mathrm{D} / \mathrm{A} \rightarrow \mathrm{D} / \mathrm{B} \mid \exists \phi \in \mathrm{D} . \forall \text { d.dAd } \Rightarrow\left(\left(\phi \mathrm{d} \downarrow \Leftrightarrow \mathrm{f}\left([\mathrm{d}]_{\mathrm{A}}\right) \downarrow\right) \wedge\left(\phi \mathrm{d} \downarrow \Rightarrow \phi \mathrm{def}\left([\mathrm{d}]_{A}\right)\right)\right\}\right.
\end{aligned}
$$

The category (per ${ }_{D}, M_{D}$ ) is a pccc. In particular the partial exponent is defined as:

$f(A \rightarrow B) g \Leftrightarrow \forall d$ e. (dAe $\left.\Rightarrow f d \cong_{B} g e\right)$.

where $t \cong_{B} s \Leftrightarrow(t \downarrow \Leftrightarrow s \downarrow)$ and $(t \downarrow \Rightarrow t B s)$ i.e. Kleene equality relativized to $B$. Notice that the category has enough points. The terminal object is any per with one equivalence class, say $1 \triangleq \mathrm{D} \times \mathrm{D}$. In this case the dominance is $\Sigma \triangleq 1 \rightarrow 1=\{1, T\}$, where: $\perp \triangleq\{\mathrm{d} \in \mathrm{D} \mid \forall \mathrm{e} . \mathrm{de} \uparrow\}, T \triangleq$ $\{d \in D \mid \forall e . d e \downarrow\}$.

\subsubsection{D-order implies intrinsic order on pers}

Applying the constructions in the previous section we can now define:

$\Sigma \mathrm{Ner}_{D}$ as the full subcategory of per $_{D}$ whose objects are $\Sigma$-objects.

What is the relationship between the D-order (2.1.3.(2)) and the intrinsic pre-order (1.2.2) ? If $d \leq_{D} d^{\prime}, A \in$ per $_{D}$, and $d, d \in|A|$ then a fortiori $[d]_{A} \leq_{A}\left[d^{\prime}\right]_{A}$. Suppose $B \in \Sigma(A)$ and $[d]_{A} \in D / B$ then there is a $W \in \Sigma(D)$ such that $|A| \cap W=|B|$. But by hypothesis $d^{\prime} \in W$ and therefore $\left[\mathrm{d}^{\prime}\right]_{\mathrm{A}} \in \mathrm{D} / \mathrm{B}$.

The sense of this remark is that if two elements cannot be separated in the type free universe of the realizability structure $D$ then a fortiori they cannot be separated in the "typed" structure of pers. An interesting approach can arise by trying to reverse this implication i.e. consider a canonical representative for each equivalence class and find conditions under which the intrinsic order on pers forces the underlying order.

\subsection{Reflection of $\Sigma$ per}

We give an elementary and direct proof that $\Sigma \operatorname{per}_{D}$ is reflective in $\operatorname{per}_{D}$. This fact can also be derived following more abstract considerations, see for example proof 4.2.6.

\subsubsection{Theorem ( 5 per $\varsigma_{>}$per)}

$\Sigma$ per is a full reflective subcategory of per.

Proof

The very simple idea for obtaining a $\Sigma$ per $L_{\Sigma}(A)$ from the per $A$ is to collapse equivalence classes that cannot be separated by $\Sigma(A)$. Given a per $A$ and the intrinsic preorder $s_{A}$ we can define, as usual, an equivalence relation, $\approx_{A}$, on $D / A$ as:

$$
[d]_{A} \approx A[e]_{A} \Leftrightarrow d, e \in|A| \wedge[d]_{A} \leq_{A}[e]_{A} \wedge[e]_{A} \leq_{A}[d]_{A} \text {. }
$$

Now define the reflector $L_{\Sigma}:$ per $\rightarrow \Sigma$ per as follows:

if $A \in$ per then $L_{\Sigma}(A)$ is such that: $d L_{\Sigma}(A) e \Leftrightarrow[d]_{A} \approx_{A}[e]_{A}$

if $f: A \rightarrow B$ then $L_{\Sigma}(f)\left([d]_{L \Sigma(A)}\right)=f\left([d]_{A}\right)$

One can easily verify that: (1) $L_{\Sigma}(A)$ is a $\Sigma$ per. Actually it is the least $\Sigma$ per containing $A$ (as a relation). (2) $d L_{\Sigma}(A) e \Rightarrow f\left([d]_{A}\right)=f\left([e]_{A}\right)$, as $B$ is separated. (3) Every map from a per $A$ to a $\Sigma$ per B can be uniquely extended to a map from $L_{\Sigma}(A)$ to $B$. From these facts it is easy to exhibit the natural isomorphism of the adjunction. 


\subsubsection{Corollary (Closure properties of 5 per) \\ $\Sigma$ per is a ccc and it has all limits and colimits of per. Proof}

The existence of limits and colimits is guaranteed by 2.3 .1 and note 1.2.5. Try, as an exercise, to show that $\Sigma$ per is closed under exponentiation.

\section{N-completeness}

One is interested in finding an analogous of the notion of $\omega$-completeness in a realizability framework. In the first place we need an object $\mathrm{N}$ that can play the role of the natural numbers. ${ }^{11}$ Next we will concentrate on the $N$-complete Epers that is those Epers such that any ascending sequence on them, that is definable as a map in the category, has a lub w.r.t. the intrinsic order.

When restricting the attention to $N$-complete Sper over Kleene's pca (2.1.4.(1)) it is possible to prove a remarkable generalization ${ }^{12}$ of a theorem in Myhill\&Shepherdson[55] asserting that all the maps preserve lubs of chains definable in the category. This will arise as a corollary of the fact that for any per $A \Sigma(\mathrm{A})$ is a collection of $(\mathrm{N}-)$ Scott's opens. The line of the proof is that of Rosolini[86] with the warning that there one works in the internal logic of the effective topos.

The remarkable application of this fact is that the full subcategory of N-complete, separated pers can be seen as a sort of pre-O-category (Wand[79], Smyth\&Plotkin[82]) in that the morphisms are partially ordered, there are lubs ${ }^{*}$ of $(\mathrm{N}-)$ chains, and the operation of composition preserves this structure.

\subsection{N-complete $\Sigma$ per $\left(c_{\mathrm{N}} \Sigma\right.$ per)}

In this section we work over an arbitrary pca $D$, however for getting the main results (3.2) additional assumptions on $D$ seem necessary. When stating the condition of completeness for a $\Sigma$ per A we will only be interested in the existence of the lowest upper bounds (lubs) of the chains, $\chi: N \rightarrow A$, that are definable as maps from the nno $N$ to $A$, briefly we will write $\chi: \mathrm{AS}(\mathrm{A})$ (AS for ascending sequence). Let us observe at once the existential content of this condition. Under a constructive reading we are not only requiring the existence of the lub of every ascending sequence but also a method to find such lub given a realizer for the sequence. Indeed as soon as we consider the problem of the closure of the collection of $\mathrm{N}$-complete objects w.r.t. the functional space constructor we realize that it is important to have a realizer that uniformly, for every ascending sequence of a given type, computes the lub. This leads to the following definition.

\subsubsection{Definition ( $N$-completeness)}

We write $\chi: \mathrm{AS}(\mathrm{A}) \quad$ iff $\quad \chi: \mathrm{N} \rightarrow \mathrm{A} \wedge \forall \mathrm{n}: \mathrm{N} \cdot\left(\chi \mathrm{n} \leq_{\mathrm{A}} \chi \mathrm{n}+1\right)$.

A Eper $A$ is $N$-complete iff $\forall \chi: A S(A) . \exists \operatorname{lub}_{A} \chi$,

where the existence of $l u b$ has to be interpreted constructively, that is:

(a) $\quad \forall \chi: A S(A) \cdot \exists x: A \cdot\left(\forall n: N \cdot\left(\chi n \leq_{A} x\right) \wedge \forall y: A \cdot\left(\forall n: N \cdot\left(\chi n \leq_{A} y\right) \Rightarrow x \leq_{A} y\right)\right.$ and

(b) $\exists \sigma_{A}: A S(A) \rightarrow A \cdot \forall \chi: A S(A) \cdot \sigma_{A}(\chi)=\operatorname{lub}_{A} \chi$.

Since the map $\sigma_{A}$, if it exists, is uniquely determined we will simply indicate with $A$ rather than with $\left(A, \sigma_{A}\right)$ a $N$-complete $\Sigma$ per (henceforth $c_{N} \Sigma$ per).

\section{Notes}

(1) Whenever we select a subset of the equivalence classes of a ( $\Sigma$ )per we can naturally

\footnotetext{
${ }^{11}$ More precisely a natural number object (nno) is a diagram $1 \rightarrow 0 \mathrm{~N} \rightarrow \mathrm{SN}_{\mathrm{N}}$ that is initial among all diagrams of the shape: $1 \rightarrow{ }^{X_{A} \rightarrow f_{A} \text {. }}$

12 See note 3.2.6.(1) for a rephrasing of the original statement of the theorem.
} 
consider it as a $(\Sigma)$ per. E.g. $A S(A)$ is a subset of $D /(N \rightarrow A)$ and $U \in \Sigma(A)$ is a subset of $D / A$.

(2) Given $N$ it is fairly easy to show that $c_{N} \Sigma$ per is a non-trivial category. For example every separated object $A$ in which all elements are incomparable is going to be in $c_{N} \Sigma$ per as one can define $\sigma_{\mathrm{A}} \triangleq \lambda x: \mathrm{AS}(\mathrm{A}) . \chi(0)$ where 0 is the zero of the nno (every ascending sequence on a flat object is constant).

\subsubsection{Definition (Scott open)}

Let $A$ be a per. $A$ subset $U$ of $D / A$ is a Scott open and we write $U \in \tau(A)$ iff

(1) $\forall x, y: A .\left(x \in U \wedge x \leq_{A} y \Rightarrow y \in U\right)$. (2) $\forall \chi: A S(A) .\left(\exists \operatorname{lub}_{A} \chi: U \Rightarrow \exists n: N .(\chi n: U)\right)$.

Note that this definition makes sense in any preorder.

\subsection{Realized maps are $\mathrm{N}$-continuous}

It is immediate to check that $\tau(\mathrm{A})$ defines a topology over A. Before stating the main result we need to look closer at the nno.

\subsubsection{Natural number object in a generic pca.}

Denote with $\underline{n}$ the term corresponding to the numeral $\mathbf{n}$ in a pca, say the compilation in a pca of Church numerals. Let $\mathrm{n}^{\mathrm{D}}$ be the interpretation of the numeral in the pca $\mathrm{D}$. We set: $\left.N_{C N} \triangleq\left\{n^{D}\right\} \mid n \in \omega\right\}$

In a pca all the basic arithmetic operations are definable on the numerals. As a consequence of this fact if $D$ is non-trivial and $n \neq m$ then $n^{D} \neq m$. Moreover $N_{C N} \in \Sigma$ per. Finally one can show that $\mathrm{N}_{\mathrm{CN}}$ is a nno in the category.

\subsubsection{Natural number object in $(\omega, \cdot)$.}

When working in $(\omega, \cdot)$ we can define as nno:

$$
\mathrm{N}_{\omega} \triangleq\{\{\mathrm{n}\} \mid \mathrm{n} \in \boldsymbol{\omega}\}
$$

In the following we will use the fact that $\{\{n\} \mid n n \uparrow\}$ does not belong to $\Sigma(N)$. In a generic pca having all the tools of arithmetic we can still express concepts like "the n-th turing machine applied at input $m$ converges in at least $k$-steps" however the problem is that it can still be the case that $\{(n) \mid n n \uparrow\}$ belongs to $\Sigma(N)$. This is not surprising as the operation of application can be completely unrelated to the gödel-numbering. For example consider the pca satisfying in the category of directed complete partial orders and partial continuous maps: $\omega+(D \rightarrow D) \triangleleft D$, where $\omega$ are the natural numbers with the flat ordering. Then

$\mathrm{N}_{\mathrm{D}} \triangleq\{\{\mathrm{d}\} \mid \mathrm{d}$ is the image of $\omega$ via embedding is a nno, however all its subsets are still semi-computable as they are Scott's open. The following theorem shows that if $A \in c_{N} \Sigma$ per $_{\omega}$ then the semi-computable predicates on A are Scott's open.

\subsubsection{Theorem $(\Sigma(A) \subseteq \tau(a))$} Proof

If $A \in c_{N} \Sigma \operatorname{per}_{\omega}$ and $U \in \Sigma(A)$ then $U \in \tau(A)$.

The first condition of upward closure follows by the definition of intrinsic order. Take $x: U$ and suppose $x \leq_{A} y$ then $y: U$ as $x \leq_{A} y$ iff $\forall U \in \Sigma(A) .(x: U \Rightarrow y: U)$.

The proof of the second condition takes advantage of the specific recursion-theoretic character of the structures we have built. In this sense the following proof is the kernel of this approach to the development of the theory. In the following: $N \equiv N_{\omega}$.

Consider the set $K \triangleq\{(n\} \mid n n \downarrow\} \subseteq D / N$. Observe $K \in \Sigma(N)$ and $K^{c} \notin \Sigma(N)$, where $K^{c}$ is $K$ complement w.r.t. N. Consider the predicate nn $\downarrow$ i meaning that the computation $n n$ of the $\mathrm{n}$-th machine applied to the input $\mathrm{n}$ will stop in at most $\mathrm{i}$ steps. This is a decidable predicate.

Now let us proceed by contradiction assuming there is $\chi: A S(A)$ such that: 
$\exists \operatorname{lub}_{\mathrm{A}} \chi: \mathrm{U}$ and $\forall \mathrm{n}: \mathrm{N} . \sim(\chi \mathrm{n}: \mathrm{U})$.

The crucial idea is to build a map $\mathrm{h}: \mathrm{N} \rightarrow \mathrm{A}$ such that, under the condition $\forall \mathrm{n}: \mathrm{N}$. $\sim(\chi n: U)$, maps $K$ in $\{\chi n \mid n: N\}$ and $K^{c}$ in $l^{n} b_{A} \chi$. Next one uses the pullback condition to conclude that $\mathrm{h}^{-1}(\mathrm{U})=\mathrm{K}^{\mathrm{c}} \in \Sigma(\mathrm{N})$.

We now define a family of chains $c(n, i): N \rightarrow(N \rightarrow A)$. Let $\mu k \leq i . n n \downarrow k$ be the least element $k$ less then $i$ s.t. $n n$ converges in $k$ steps. Define

$$
c(n, i) \triangleq \text { if } \sim(n n \downarrow i) \text { then } \chi \mathrm{i} \text { else } \chi(\mu \mathrm{k} \leq \mathrm{i} . \mathrm{nn} \downarrow \mathrm{k})
$$

Observe that for any given $n$ if $n \in K$ then $c(n, i)$ coincides with the ascending sequence $\chi$ up to the first $k$ s.t. $n n \downarrow k$ and then becomes definitely constant; on the other hand if $n \in K^{c}$ then $c(n, i)$ coincides with $\chi$.

We use the existence of a map that uniformly realizes the lub of ascending sequences to define the map $h: N \rightarrow A$ as follows:

$$
h(n) \triangleq \sigma_{A}(\lambda i . c(n, i)) \text {. }
$$

As we have just observed $h(n)=\operatorname{lub}_{A} \chi$ if $n \in K^{c}$ and $h(n) \in\{\chi n \mid n: N\}$ o.w. .

From this we can conclude $h^{-1}(U)=K c \in \Sigma(N)$ obtaining the desired contradiction.

For the reader logically inclined let us mention how this proof by contradiction can be turned into a "constructive proof" via Markov Principle that is stated as follows (see Troelstra\&vanDalen[88], vol. I):

$$
\forall \mathrm{n}: \mathrm{N} \text {.(Rn } \vee \neg \mathrm{Rn}) \wedge \neg \neg \exists \mathrm{n}: \mathrm{N} .(\mathrm{Rn}) \rightarrow \exists \mathrm{n}: \mathrm{N} \text {.(Rn). }
$$

This is often rephrased as follows: if we have an effective way of testing the predicate $R$ and if the non-existence of an element satisfying $R$ leads to an absurd then there is an element satisfying $R$ (that can be effectively found by an enumeration technique). If the predicate is just semi-decidable like in the case $\chi n: U$ then the principle can be easily adapted. In our case we proved $\neg \neg \exists$ n:N.( $\chi n: U)$ from which we can conclude $\exists n: N .(\chi n: U)$.

\subsubsection{Definition ( $N$-chains preservation)}

Let $\mathrm{A}, \mathrm{B} \in \Sigma$ per. Then we say that $\mathrm{f}: \mathrm{A} \rightarrow \mathrm{B}$ preserves $N$-chains iff $\forall \chi: A S(A) .\left(\exists \operatorname{lub}_{A} \chi: A \Rightarrow\left(\exists \operatorname{lub} b_{B} f \chi: B \wedge f\left(l u b_{A} \chi\right)=l u b_{B} f \chi\right)\right)$.

\subsubsection{Proposition (Realized maps are Scott continuous)}

Suppose $A, B \in \mathrm{c}_{\mathrm{N}} \Sigma$ per $_{\omega}$.

(1) Any map f: $A \rightarrow B$ preserves $N$-chains.

(2) If $\mathrm{f}: \mathrm{A} \rightarrow \mathrm{B}$ preserves $\mathrm{N}$-chains then it is (N-)Scott continuous.

Proof

(1) Consider $\chi$ : AS(A) and assume $\exists \exists_{\mathrm{Aub}} \chi$ :A. In order to show $\exists \mathrm{lub}_{\mathrm{B}} \mathrm{f} \chi=\mathrm{f}\left(\mathrm{lub}_{\mathrm{A}} \chi\right)$ we prove that for any majorant $y: B$ of $f \chi: A S(B)$ we have $f\left(l_{u b} \chi \chi\right) \leq_{B} y$.

We recall that $f\left(\operatorname{lub}_{A} \chi\right) \leq_{B} y$ iff $\forall U \in \Sigma(B)$. (f(lub $\left.\left.A \chi\right): U \Rightarrow y: U\right)$. Now $U \in \Sigma(B)$ implies by the pullback condition of admissible domains: $\mathrm{f}^{-1}(\mathrm{U}) \in \Sigma(\mathrm{A})$ i.e. by the previous theorem $f^{-1}(U) \in \tau(A)$. Since $f\left(l u b_{A} \chi\right): U$ we have $\operatorname{lub}_{A} \chi: f^{-1}(U)$ that implies by the definition of open set $\exists n: N .\left(\chi n: f^{-1}(U)\right)$. Therefore $\exists n: N .(f \chi n: U)$ and this implies $y: U$.

(2) As usual take $U \in \tau(B)$ and consider $f^{-1}(U)$. This is upward closed by the fact that $f$ is monotone. Moreover consider $\chi: A S(A)$ and suppose $\exists \operatorname{lub}_{A} \chi: f^{-1}(U)$. Then by hypothesis $f\left(l u b_{A} \chi\right)=l u b_{B} f \chi: U$. Therefore $\exists n: N .(f \chi n: U)$ i.e. $\chi n: f^{-1}(U)$.

\subsubsection{Notes}

(1) On Myhill and Shepherdson theorem. Suppose $\left\{\mathrm{E}_{\mathrm{n}}\right\}_{\mathrm{n} \in \omega}$ is an enumeration of finite subsets of $\omega$ and $<,>: \omega \times \omega \rightarrow \omega$ is a pairing map. Given $X, Y \in 2^{\omega}$ define:

$$
X \cdot Y \triangleq\left\{m \mid \exists n .\left(<n, m>\in X \wedge E_{n} \subseteq Y\right)\right\}
$$

The reader may recognize in this definition the notion of application that arises in graph 
models. Denote with RE the collection of recursively enumerable sets. Every $W \in R E$ determines a functional $F_{W}: R E \rightarrow R E$ that is defined as:

$F_{W}(X) \triangleq W \cdot X$

Such functionals are called enumeration operators (or also recursive functionals). It is immediate to check that these functionals are continuous w.r.t. the order given by setcontainment.

Next observe that RE can be seen as a totally enumerated set and therefore as a total equivalence relation. In particular it can be identified with $\operatorname{Id}_{\omega} \rightarrow 1$ where: $\mathrm{nId}_{\omega} \mathrm{m} \Leftrightarrow \mathrm{n}=\mathrm{m}$ and $\forall \mathrm{n}, \mathrm{m} . \mathrm{n} 1 \mathrm{~m}$. Note that the intrinsic order on $\mathrm{Id}_{\omega}-1$ coincides with the one given by set-containment.

Myhill-Shepherdson theorem can then be stated as follows:

$F \in \operatorname{per}_{\omega}[R E, R E] \Leftrightarrow F$ is an enumeration operator

This was intended as a characterization of certain "type-2" functionals which are called effective operations and which correspond exactly to the morphisms in per ${ }_{\omega}[\mathrm{RE}, \mathrm{RE}]$. The interesting part of the proof consists in showing that the effective operations are enumeration operators and, therefore, are continuous. This is the sense that is generalized in 3.2.5.(2).

(2) On the Effectivity of the Interpretation.Consider the definition of the exponential: $\mathrm{fAA}_{\mathrm{g}} \Leftrightarrow \forall \mathrm{n}, \mathrm{m}$. nAm $\Rightarrow \mathrm{fnAgm}$. Since A occurs both positively and negatively it is immediate to observe that the logical complexity of $\mathrm{A}^{\mathrm{A}}$ is greater than that of $\mathrm{A}$. In fact it is easy to build examples in which starting with a decidable relation, say $\mathrm{Id}_{\omega}$ and iterating the exponentiation operation one can reach any level of the arithmetic hierarchy. Therefore the problem arises of the "effectivity" of this class of models. ${ }^{13}$ It may be the case that the category considered here is too large and that further conditions of "algebraicity" have to be imposed.

\section{E-connection and Extensionality}

In 4.1, following Phoa[90], we introduce the notion of $\Sigma$-connected object. It turns out that on $\Sigma$-connected objects the intrinsic order on a product coincides with the pointwise order. Moreover every $c_{N} \Sigma$ per over $(\omega, \cdot)$ is $\Sigma$-connected. Hence we can regard the intrinsic order of products existing in $\mathrm{C}_{\mathrm{N}} \Sigma$ per $_{\omega}$ as pointwise.

The quest for a realizer that, uniformly, for every object and for every ascending sequence on that object, computes the lub can be seen as the principal motivation for the invention of the full subcategory of extensional pers (exper), proposed in Freyd\&al.[90] (4.2). It turns out that exper ${ }_{D}$ is reflective in $\operatorname{Lper}_{D}$ and that, moreover, the category of $\mathrm{N}$ complete extensional pers over $\left(\omega_{i}\right)\left(c_{N} \operatorname{exper}_{\omega}\right)$ is reflective in exper ${ }_{\omega}$ (4.3). Finally we show that there is an exper ${ }_{\omega}$ that is not isomorphic to any $\Sigma$ per $_{\omega}(4.4)$.

\subsection{Intrinsic Ordering vs. Pointwise Ordering}

Let $x, y: \prod_{i \in I} A_{i}$ be two points of an indexed product in per $_{D}$.

We say that $x$ is pointwise-less than $y$ iff $\forall i \in I . \pi_{j} x \leq_{A i} \pi_{i} y$.

Observe that if $x \leq y$ in the intrinsic order then, by the monotonicity of the projections, $x$ is pointwise-less than $y$. Under which conditions does the point-wise order imply the intrinsic order?

Let us start by re-examining the properties of $\Sigma$. We recall (see 2.2.4) that if we take $1=D \times D$ then $\Sigma \triangleq 1 \rightarrow 1$ is composed of two equivalence classes that of total elements and that of always divergent elements. We write $D / \Sigma=\{\perp, T\}$. It is easy to see that $\neg\left(1 \leq_{\Sigma} T\right)$ (hence $\Sigma$ is separated). In the following we assume to work over $(\omega, \cdot)$. Then it is also possible to show:

$\overline{13}$ Compare the situation with what happens in standard domain theory. There the interpretations can be effectively enumerated and the notions of approximation and equivalence of two terms are in $\Pi_{2}{ }^{0}$. 
$1 \leq_{\Sigma} T$ (see 4.4.1 for a recursion-theoretic argument).

Observe that given $x, y: A$, if there is $f: \Sigma \rightarrow A$ such that $f \perp=x$ and $f T=y$ then we can conclude $x \leq_{A} y$ by the monotonicity of $f$. Moreover such map $f$, if it exists, is unique. The idea is to show that for $x, y: \prod_{i \in I} A_{i}$ in the relation given by the pointwise order we can construct a map $f$ that proves that they are in the relation of the intrinsic order.

\subsubsection{Definition ( $\Sigma$-connection) $)^{14}$}

We say that $A$ is $\Sigma$-connected iff $\quad \forall x, y: A .(x \leq A y \Rightarrow \exists f: \Sigma \rightarrow A .(f \perp=x \wedge f T=y)$.

It has been observed by W. Phoa that $\Sigma$-connection is a property that enjoys good closure properties and forces the coincidence of pointwise and intrinsic orders on products.

\subsubsection{Proposition ( $\Sigma$ connection is preseroed by arbitrary products)}

If $\forall i \in I A_{i}$ is $\Sigma$-connected and if it exists the product, $\prod_{i \in I} A_{i}$, then such product is $\Sigma$ connected. Moreover if $\mathrm{D} \equiv(\omega, \cdot)$ then the intrinsic order coincides with the pointwise order. Proof

First an elementary remark: if $x, y: B \rightarrow \prod_{i \in I} A_{i}$ and $\forall i \in I . \pi_{i} x=\pi_{i} y$ then $x=y$. Next consider $x, y: \prod_{i \in I} A_{i}$ such that $\forall i \in I . \pi_{i} x \leq_{A i} \pi_{i} y$. By hyp. $\forall i \in I . \exists f_{i}: \Sigma \rightarrow A_{i} \cdot\left(f_{i} \perp=\pi_{i} x \wedge f_{i} T=\pi_{i} y\right)$. By def. of product we have a map $\left\langle\mathrm{f}_{\mathrm{i}}\right\rangle: \Sigma \rightarrow \prod_{i \in I} A_{i}$ such that $\left.\pi_{\mathrm{i}}<\mathrm{f}_{\mathrm{i}}\right\rangle=\mathrm{f}_{\mathrm{i}}$. But this forces:

$\forall \mathrm{i} \in \mathrm{I} .\left(\pi_{\mathrm{i}}<\mathrm{f}_{\mathrm{i}}>\perp=\mathrm{f}_{\mathrm{i}} \perp=\pi_{\mathrm{i}} \mathrm{x} \wedge \pi_{\mathrm{i}}<\mathrm{f}_{\mathrm{i}}>\mathrm{T}=\mathrm{f}_{\mathrm{i}} \mathrm{T}=\pi_{\mathrm{i}} \mathrm{y}\right)$. By the initial remark: $\left\langle\mathrm{f}_{\mathrm{i}}>\perp=\mathrm{x} \wedge\left\langle\mathrm{f}_{\mathrm{i}}\right\rangle \mathrm{T}=\mathrm{y}\right.$. Hence the product is $\Sigma$-connected. Finally observe that in this proof we only used the fact that $x$ is pointwise less than $y$, hence the map $\left\langle f_{i}>\right.$ proves that $x \leq_{A} y$.

\subsubsection{Corollary}

Let $\mathrm{A}, \mathrm{B}$ be pers over a pca $\mathrm{D}$, and $\Sigma$ the dominance.

(1) $\Sigma$ and $\Sigma^{B}$ are $\Sigma$-connected.

(2) If $B$ is $\Sigma$-connected and $D / A \subseteq D / B$ then $A$ is $\Sigma$-connected. Moreover the intrinsic order on $A$ coincides with the restriction of the intrinsic order on $B$.

Proof

(1) That $\Sigma$ is connected is obvious. $\Sigma^{B}$ can be seen as a degenerate case of product, therefore we can apply 4.1.2.

(2) In general if $x \leq_{A} y$ then $x \leq_{B} y$, indeed every map $f: B \rightarrow \Sigma$ can be restricted to a map $f: A \rightarrow \Sigma$. Vice versa take $x, y: A$. If $x \leq_{B} y$ then we can consider the connecting map $f: \Sigma \rightarrow B$ such that $f \perp=x \wedge f T=y$. Now the related connecting map $f^{\prime}: \Sigma \rightarrow A$ obtained by restricting the codomain of $f$ proves that $x \leq_{A} y$.

In the case $\mathrm{D} \equiv(\omega, \cdot)$ one can prove that every $\mathrm{N}$-complete $\Sigma$-per is $\Sigma$-connected.

\subsubsection{Proposition (every $c_{N} \Sigma p e r_{\omega}$ is $\Sigma$-connected)}

\section{Proof}

Let $D \equiv(\omega, \cdot)$. If $A$ is in $c_{N} \Sigma$ per $_{\omega}$ then $A$ is $\Sigma$-connected.

The proof does not use at all the fact that we have lubs of arbitrary chains but rather the less obvious fact that lubs can be effectively computed. Let $x \leq_{\mathrm{A}} \mathrm{y}$ and consider the following family of chains: $\mathrm{c}(\mathrm{n}, \mathrm{i}) \triangleq$ if $\mathrm{nn} \downarrow \mathrm{i}$ then $\mathrm{y}$ else $\mathrm{x}$

Fixed $\mathrm{n}$ the ascending sequence $\lambda \mathrm{i}$.c( $\mathrm{n}, \mathrm{i})$ is apparently innocuous because it can take at most two values, however in general we have no tool to compute the lub as we do not know a priori how far in the sequence to look.

We use the existence of the map $\sigma_{\mathrm{A}}: \mathrm{AS}(\mathrm{A}) \rightarrow \mathrm{A}$ to define a map $\mathrm{h}:\left[\mathrm{Kc}^{\mathrm{c}}, \mathrm{K}\right\} \rightarrow \mathrm{A}$ such that:

\footnotetext{
14This is the "external analogous" of Phoa's notion of linked E-space. Warning: there is no direct relation to the topological notion of connection.
} 
$h(K c)=x, h(K)=y \quad\left(K\right.$ and $K^{c}$ are defined as in 3.2.3). Next observe that it is possible to define an iso realized by the identity: $h: \Sigma \rightarrow\left\{K^{c}, K\right\}$. Hence by composition we obtain the desired map.

\subsubsection{Corollary (Closure properties)}

$c_{N} \sum_{\text {per }}$ is cartesian closed and it has all finite limits.

\section{Proof}

Use the fact that the intrinsic order is pointwise to define the lub of a chain of maps. One can verify that equalizers preserve $\mathrm{N}$-completeness.

\subsection{Extensional PER (exper)}

In this section, following work by Freyd\&al.[90], we introduce the notion of extensional per (exper). Extensional pers are $\Sigma$-connected and they form a full reflective subcategory of इpers.

\subsubsection{Intuition}

What is a per? The question may seem rethoric in that our intuition so far has been that pers are data types. The data in the per $\mathrm{A}$ are preordered by a collection of legal observations or tests that are represented by the maps from $A$ into the dominance $\Sigma$. We would like to emphasize this point of view by setting:

$\operatorname{Test}(\mathrm{A}) \triangleq \mathrm{A} \rightarrow \Sigma$.

On the other hand we could ask if a per can be seen as a collection of tests on a data type (i.e. a per). We will call such a per extensional. Towards this purpose define a per of invariants for $A$, say $\operatorname{Inv}(A)$, as follows:

$\operatorname{dInv}(A) e \Leftrightarrow \forall f, g . f A g \Rightarrow f d \cong g e$

The intuition is that Inv(A) is the greatest per for which $A$ can be seen as a collection of functions on it. The following proposition supports this idea. We will take the assumption of 4.2.2.(4) as definition of exper (4.2.3).

\subsubsection{Proposition (on Test and Inv)}

Let $\mathrm{A}$ be a per over a pca $\mathrm{D}$. Then:

(1) $A \subseteq \operatorname{Inv}(\operatorname{Test}(A))$.

(2) $A \subseteq \operatorname{Test}(\operatorname{Inv}(A))$.

(3) If $D / A \subseteq D / \operatorname{Inv}(\operatorname{Test}(A))$ then $A$ is separated.

(4) If $D / A \subseteq D /(B \rightarrow \Sigma)$ then $D / A \subseteq D / \operatorname{Test}(\operatorname{Inv}(A))$.

Proof

(1) Note: dinv(B)e $\Leftrightarrow \lambda$ u.ud $(B \rightarrow \Sigma) \lambda$ u.ue. Moreover:

$\mathrm{dAe} \Rightarrow \lambda \mathrm{u}$.ud $(\mathrm{A} \rightarrow \Sigma) \rightarrow \Sigma \lambda$ u.ue, hence by instantiating $\mathrm{B}$ with $\mathrm{A} \rightarrow \Sigma$ we get $A \subseteq \operatorname{Inv}(T e s t(A))$.

(2) By definition $f \operatorname{Test}(\operatorname{Inv}(A)) g$ iff $f \operatorname{Inv}(A) \rightarrow \Sigma g$. But suppose $f A g$ and $\operatorname{dInv}(A)$ e then, by definition of $\operatorname{Inv}(A)$, fdige.

(3) In general if $A \subseteq B$ then $D / A \subseteq D / B$ iff $\forall d \in|A|$.(dBe $\Rightarrow d A e)$. A $E$ per iff $\forall$ d, e $\in|A|$. ( $\lambda$ u.ud $(A \rightarrow \Sigma) \rightarrow \Sigma \lambda$ u.ue $\Rightarrow$ dAe $)$. But by hyp.

$\forall \mathrm{d} \in|\mathrm{A}|$. dInv(Test(A))e $\Rightarrow \mathrm{dAe}$, and $\operatorname{dInv}($ Test(A))e $\Leftrightarrow \lambda$ u.ud $(A \rightarrow \Sigma) \rightarrow \Sigma \lambda$ u.ue.

(4) First observe that if $D / A \subseteq D /(B \rightarrow \Sigma)$ then $B \subseteq \operatorname{Inv}(A)$. From which follows: $\operatorname{Test}(\operatorname{Inv}(A)) \equiv \operatorname{Inv}(A) \rightarrow \Sigma \subseteq(B \rightarrow \Sigma)$.Together with $(2)$ we obtain: $A \subseteq \operatorname{Test}(\operatorname{Inv}(A)) \subseteq(B \rightarrow \Sigma)$. Finally observe: $\forall \mathrm{d} \in|A|$. $d \operatorname{Test}(\operatorname{Inv}(A)) e \Rightarrow d(B \rightarrow \Sigma) e \Rightarrow d A e$.

\subsubsection{Definition (exper)}

A per is extensional iff there is a per $B$ such that: $D / A \subseteq D /(B \rightarrow \Sigma)$. 


\subsubsection{Proposition (basic properties of exper)}

In the following let $A, B$ be pers over a pca $D$, and $\Sigma$ the dominance.

(1) If $A$ is extensional then $A$ is separated and $\Sigma$-connected.

(2) If $A$ is extensional, say $D / A \subseteq D /(B \rightarrow \Sigma)$, then the intrinsic order on $A$ coincides with the restriction of the intrinsic order on $B \rightarrow \Sigma$ which, if $D \equiv\left(\omega,{ }^{*}\right)$, coincides with the pointwise order. That is, given $A$ exper, $f, g: A$ we have:

$$
\mathrm{f} \leq_{\mathrm{A}} \mathrm{g} \Leftrightarrow \mathrm{f} \leq_{\mathrm{B} \rightarrow \Sigma \mathrm{g}} \Leftrightarrow \forall \mathrm{b}: \mathrm{B} . \mathrm{fb} \leq_{\Sigma} \mathrm{gb} \text {. }
$$

(3) If $B$ is extensional and $D / A \subseteq D / B$ then $A$ is extensional.

(4) exper is closed under arbitrary intersections.

\section{Proof}

(1) From 1.2.4 we know that $\Sigma^{B}$ is separated and $\Sigma$ per is closed under subobjects. From 4.1.3(1) we know that $\Sigma^{B}$ is $\Sigma$-connected and that the $\Sigma$-connected objects are closed under subobjects obtained by selecting a subset of the quotient space.

(2) $\mathrm{f} \leq_{\mathrm{A}} g \Leftrightarrow \mathrm{f} \leq_{\mathrm{B} \rightarrow \Sigma \mathrm{I}} \mathrm{g}$ follows from 4.1.3.(2). $\mathrm{f} \leq_{\mathrm{B} \rightarrow \Sigma} g \Leftrightarrow \forall \mathrm{b}: \mathrm{B}, \mathrm{fb} \leq_{\Sigma} \mathrm{gb}$ follows from 4.1:2.

(3) Immediate.

(4) Suppose $D / A_{i} \subseteq D /\left(B_{i} \rightarrow \Sigma\right)$ for $i \in I$. Then $D / \cap_{i \in I} A_{i} \subseteq D /\left(\cup_{i \in I} B_{i} \rightarrow \Sigma\right)$.

\subsubsection{Remark (comparison with Freyd\&al.[90])}

The notion of extensional per in Freyd\&al.[90], say ex', can be stated as follows: $A$ is an ex'per iff there is a set $X \subseteq D$ such that:

$\mathrm{D} / \mathrm{A} \subseteq \mathrm{D} /(\operatorname{Diag}(\mathrm{X}) \rightarrow \Sigma) \quad$ where $\operatorname{dDiag}(X)$ e iff $\mathrm{d}=\mathrm{e} \in \mathrm{X}$.

Notice that $(\operatorname{Diag}(X) \rightarrow \Sigma)$ is always a total equivalence relation. However the two definitions are equivalent. Obviously any ex'per is an exper. For the vice versa observe that $\mathrm{D} /(\mathrm{B}-1) \subseteq \mathrm{D} /(\operatorname{Diag}(|\mathrm{B}|)-1)$.

\subsubsection{Theorem (exper $D \subset_{>}$Eper $\left._{D}\right)$}

Let $D$ be a pca, then exper $D$ is a full and reflective subcategory of $\Sigma$ per $_{D}$. Proof (after Freyd\&al.[90])

The existence of an inclusion functor follows from 4.2.4.(1). We define the reflector $L_{\text {ex }}:(\Sigma)$ per $\rightarrow$ exper as follows: $D / L_{e x}(A) \triangleq\left\{[\lambda u . u d]_{(A \rightarrow \Sigma) \rightarrow \Sigma} / \mathrm{d} \in|A|\right\}$.

This is an exper as $D / L_{e x}(A) \subseteq(A \rightarrow \Sigma) \rightarrow \Sigma$. The universal map $e_{A}: A \rightarrow L_{e x}(A)$ is the one realized by $\lambda$ du.ud. Intuitively it takes an element $d$ to the collection of its neighborhoods $\lambda u . u d$. By construction $e_{A}$ is an epi, moreover it is also a mono if $A$ is separated. In general $\mathrm{L}_{\mathrm{ex}}$ can also work as a reflection from per to exper.

Next we intend to show that if $B$ is extensional then $e_{B}: B \rightarrow L_{e x}(B)$ is an iso. Suppose: $D / B \subseteq D /(C \rightarrow \Sigma)$. We will take $e_{B}{ }^{-1}: L_{e x}(B) \rightarrow B$ as the map realized by: $\lambda i . \lambda c . i(\lambda u$.uc). Let us try to assign a type to this term. First check that we can write:

$$
\lambda_{i}(\mathrm{~B} \rightarrow \Sigma) \rightarrow \Sigma . \lambda c^{C} \cdot i\left(\lambda u^{B} \cdot u c\right):((B \rightarrow \Sigma) \rightarrow \Sigma) \rightarrow(C \rightarrow \Sigma) \text {. }
$$

Since $B \subseteq(C \rightarrow \Sigma)$ in the application we can "coerce" $u$ from $B$ to $C \rightarrow \Sigma$. Since $L_{\text {ex }}(B) \subseteq(B \rightarrow \Sigma) \rightarrow \Sigma$ we can also assign to the same term the type $L_{e x}(B) \rightarrow(C \rightarrow \Sigma)$. Finally we derive the type-assignment: $\lambda i . \lambda c . i(\lambda u . u c): L_{e x}(B) \rightarrow B$. Recalling the definition of $L_{e x}(B)$ we know for $f, f^{\prime} \in|B|$ :

$$
\mathrm{fBf} \Leftrightarrow \lambda v . v f L_{\mathrm{ex}}(B) \lambda v . v f^{\prime} \text {. }
$$

Assume: $\lambda v$.vf $L_{e x}(B) \lambda v . v f$ and $c C c^{\prime}$. Since $B \subseteq(C \rightarrow \Sigma)$ we derive from the former: $\lambda v . v f$ $((C \rightarrow \Sigma) \rightarrow \Sigma) \rightarrow \Sigma \lambda v . v f$. From the latter follows:

$\lambda$ u.uc $(C \rightarrow \Sigma) \rightarrow \Sigma \lambda$ u.uc'. Hence after application we reduce the problem to $f_{C} \cong f^{\prime} c^{\prime}$, that holds by the assumption $\mathrm{fBf}^{\prime}$.

As for showing inversions: 
$(\lambda i \cdot \lambda c . i(\lambda u \cdot u c))((\lambda d w \cdot w d) f)=(\lambda i \cdot \lambda c . i(\lambda u \cdot u c))(\lambda w \cdot w f)=\lambda c \cdot(\lambda w \cdot w f)(\lambda u \cdot u c)=\lambda c . f c$. Vice versa: $(\lambda d w . w d)((\lambda i . \lambda c . i(\lambda u . u c))(\lambda v . v f))=(\lambda d w . w d)(\lambda c .(\lambda v . v f)(\lambda u . u c))=$ $=(\lambda d w . w d)(\lambda c . f c)=(\lambda w . w(\lambda c . f c))$. And one observes that $\lambda c . f c C \rightarrow \Sigma f$.

It is usual to define a functor from $(A \rightarrow \Sigma) \rightarrow \Sigma$ to $(B \rightarrow \Sigma) \rightarrow \Sigma$. Given $\phi \in|A \rightarrow B|$ follow this pattern to define $\phi^{\prime} \in\left|L_{e x}(A) \rightarrow L_{e x}(B)\right|$ as follows: $\phi^{\prime} \triangleq \lambda i . \lambda u$.i $(\lambda a . u(\phi a))$. If $f=[\phi]_{A} \rightarrow B$ set $\mathrm{L}_{\mathrm{ex}}(\mathrm{f}) \triangleq\left[\phi^{\prime} \mathrm{L}_{\mathrm{ex}}(\mathrm{A}) \rightarrow \mathrm{L}_{\mathrm{ex}}(\mathrm{B})\right.$.

\subsection{Reflection of $\mathrm{N}$-complete exper $\left(\mathrm{c}_{\mathrm{N}}\right.$ exper)}

Suppose A extensional and $f, g$ : A. From 4.2.4.(4) we know that we can look at $f$ and $g$ as functions on some domain so that $\mathrm{f}_{\mathrm{A}} \mathrm{g}$ iff the domain of definition of $\mathrm{f}$ is smaller than that of $g$. This resembles very much the usual order on recursively enumerable sets seen as domains of partial recursive functions. We may then remember that on such orders we know how to compute effectively the lub of an effectively given chain; namely we return the function that on a given input converges exactly when one of the elements of the chain converges. This analogy leads to the following

\subsubsection{Theorem (cNexper $\left.\subset_{\omega} \subset_{>} \operatorname{exper}_{\omega}\right)$}

(1) There is a realizer that uniformly for every $\mathrm{N}$-complete extensional per computes the lub of ascending sequences:

$$
\exists \sigma \in \omega . \forall A \in c_{N} \operatorname{exper}_{\omega}, \forall \chi: A S(A), \forall \phi \Vdash A \chi . \sigma \phi \Vdash A l u b A \chi
$$

(2) $c_{N}$ exper $r_{\omega}$ is closed under arbitrary intersections.

(3) $c_{N}$ exper $\omega_{\omega}$ is reflective in exper.

Proof (after Freyd\&al..l90I)

(1) Suppose $A$ extensional i.e. $D / A \subseteq D / B \rightarrow \Sigma$, for some $B$. Let $\chi$ : $A S(A)$, then we have: $\forall \mathrm{n}: \mathrm{N} \cdot \chi(\mathrm{n}) \leq_{\mathrm{A}} \chi(\mathrm{n}+1)$ that is: $\forall \mathrm{n}: \mathrm{N} \cdot \forall \mathrm{b}: \mathrm{B} \cdot \chi(\mathrm{n})(\mathrm{b}) \leq \chi(\mathrm{n}+1)(\mathrm{b})$.

Now consider the map: $Y_{A} \triangleq \lambda \chi: A S(A) \cdot \lambda b: B$. if $(\exists n: N \cdot \chi(n)(b) \downarrow)$ then $\downarrow$.

It is fairly obvious how to find a realizer for this map, namely:

$\sigma \triangleq \lambda \gamma . \lambda \mathrm{d}$. if $(\exists \mathrm{n} . \gamma(\mathrm{n})(\mathrm{d}) \downarrow)$ then $\downarrow$

Given the existence in the model of $Y_{A}$ it is easy to check that $Y_{A}(\chi)=\operatorname{lub}_{A} \chi$. Moreover one observes that $\sigma$ does not depend on $\mathrm{A}$.

(2) In 4.2.4.(4) we showed that exper is closed under intersections. In particular suppose: $D / A_{i} \subseteq D /\left(B_{i} \rightarrow \Sigma\right)$ for $i \in I$. Then: $D / \cap_{i \in I} A_{i} \subseteq D /\left(\cup_{i \in I} B_{i} \rightarrow \Sigma\right)$. Next consider $\chi: A S\left(\cap_{i \in I} A_{i}\right)$,

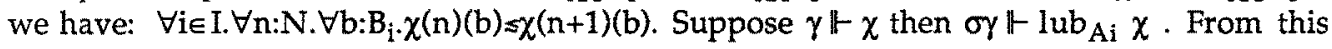
one concludes: oy lub $_{\mathrm{A}} \chi$

(3) One defines a reflector $\mathrm{L}_{\mathrm{CN}}$ : exper $\rightarrow \mathrm{c}_{\mathrm{N}}$ exper. First a remark:

$\forall \mathrm{i} \in \mathrm{I} . \mathrm{A}_{\mathrm{i}} \in \mathrm{C}_{\mathrm{N}}$ exper $\Rightarrow \exists \Pi_{\mathrm{I}} \mathrm{A}_{\mathrm{i}} \in$ exper $\Rightarrow \Pi_{\mathrm{I}} \mathrm{A}_{\mathrm{i}}$ has pointwise order $\Rightarrow$ $\Rightarrow$ using $\sigma$ we can find the lub of any ascending sequence on $\Pi_{I} A_{i} \Rightarrow \Pi_{I} A_{i} \in c_{N}$ exper. Hence $c_{N}$ exper is closed under products.

Suppose $D / A \subseteq D /(B \rightarrow \Sigma)$. Define $L_{c N}(A)$ as the least $c_{N}$ exper such that:

$D / A \subseteq D / L_{C N}(A) \subseteq D /(B \rightarrow \Sigma)$.

This is well defined because $B \rightarrow \Sigma$ is an N-complete exper. In other terms $A$ is a partial order that is the restriction of the complete order $B \rightarrow \Sigma$. If we have a chain in $A$ whose lub is not in $A$, but only in $B \rightarrow \Sigma$, then we add such point. The behavior of a map on such points is determined by $A$ hence given $\phi \Vdash f: A \rightarrow B, A$ exper and $B c_{N}$ exper one takes:

$$
\mathrm{L}_{\mathrm{CN}}(\mathrm{f}) \triangleq[\phi]_{\mathrm{LNN}_{\mathrm{N}}(\mathrm{A}) \rightarrow \mathrm{B}}
$$

\subsection{Separation vs. Extensionality}

In Phoa[90] it is observed that there is a $\Sigma \mathrm{per}_{\omega}$ that is not $\Sigma$-connected. Since the property of being $\Sigma$-connected is closed under isomorphism and since every extensional per is $\Sigma-$ connected we conclude that there is a $\Sigma \operatorname{per}_{\omega}$ that is not isomorphic to any exper. In 
particular this implies that the inclusion functor from exper ${ }_{\omega}$ to $\Sigma p r_{\omega}$ is not an equivalence. The proof points-out (yet another) surprising property of the structure: there can be two separated pers $A=\{K c, K\}$ and $B=\left\{X^{c}, X\right]$ each one with two points so that $K^{c} \leq_{A} K$, $X{ }^{c} \leq_{B} X$ and the only maps from $A$ to $B$ are the constant ones. That is there can be two domains with the same order as Sierpinski space that are not isomorphic !

We recall some basic notions of recursion theory (see, e.g., Soare[87]). Let us denote with $X$, $Y$ subsets of $\omega$, and with $X c, Y^{c}$ their complements. $X$ is m-reducible to $Y$, written, $X \leq_{m} Y$ iff $\exists f$ total recursive. $(x \in X \Leftrightarrow f(x) \in Y)$. The set $K \triangleq\{n \mid n n \downarrow\}$ is $\leq_{m}$-complete. Moreover there is a set $X$ that is not $m$-complete (see Soare[87], V.1.3). From this one proves the following list of elementary facts:

\subsubsection{Proposition}

(1) If $X$ is r.e., non-recursive, then $A \stackrel{A}{a}(X c, X)$ is a $\Sigma$ per $_{\omega}$ where $X^{c} \leq{ }_{A} X$.

(2) $\left\{K^{c}, K\right\} \equiv\{\perp, T\} \equiv \Sigma$.

(3) Let $X, Y \subset \omega$. $X \leq_{m} Y$ iff $\exists h:\{X c, X\} \mapsto\{Y c, Y\}$ mono such that $h(X)=Y$.

(4) For any $X$ r.e. there is $h:\{X c, X\} \mapsto\{K c, K\}$ mono such that $h(X)=K$.

(5) There is an $X$ r.e. such that $\{X c, X\}$ is not $\Sigma$-connected.

(6) There is a $\Sigma$ per $_{\omega}$ that is not isomorphic to any exper $\omega$. Proof

(1) A partial map from $[X c, X]$ to the terminal object cannot converge on $X^{c}$ and diverge on $X$ without contradicting the fact that $X^{c}$ is not r.e. .

(2) From $\{K c, K\}$ to $\{\perp, T\}$ consider the map realized by $\lambda n, \lambda m . n n$. In the other direction consider the map realized by the identity.

(3) Immediate from the definitions.

(4) From the fact that $\mathrm{K}$ is $\mathrm{m}$-complete and (3).

(5) From the existence, mentioned above, of an r.e. non recursive set to which $\mathrm{K}$ is not m-reducible.

(6) Recall every exper is $\Sigma$-connected, $\Sigma$-connection is invariant under isomorphisms.

\section{D-completeness and Uniformity}

The deeper results obtained so far, namely the fact that computable subsets are Scott's opens (3.4) and the existence of a uniform realizer for the lub of ascending sequences on extensional pers (4.3.1) are based on the realizability structure $(\omega, \cdot)$.

On one hand $(\omega, \cdot)$ is a very fundamental structure as it embodies the classical notion of effective computation. Moreover it is possible to build from it a very interesting model, namely the effective topos, for higher order logic enriched with principles like Church Thesis, Markov Principle, Countable Axiom of Choice, Uniformity Principle, etcetera.

On the other hand $(\omega, \cdot)$ is a highly unstructured model of computation in which we heavily use all the "coding tricks" of elementary recursion theory as exemplified by the proof of 3.4. One may wonder if it is not more reasonable towards the aim of modelling the high level constructs of programming languages to consider a more structured pca in which the basic properties of computability are abstracted in terms of order-theoretic notions and the (first order) data types have a direct and explicit correspondence at the level of the realizability structure. Before going into a formalization of this approach we should point-out that it is not clear if, in this case, an internal approach could be carried out successfully.

\subsection{D-complete, $\Sigma$ per ( $c_{D} \Sigma$ per)}

We introduce the notion of D-completeness which refers to the refinement pre-order of the pca (2.1.3.(2)) rather than to the intrinsic pre-order. 


\subsubsection{Assumption on the pca: $D_{\text {dcpo }}$}

Assume that $\mathrm{D} \rightarrow \mathrm{D} \triangleleft \mathrm{D}$ in the category of directed complete partial orders and partial continuous maps (dcpo). Then $\left(\mathrm{D}, \mathrm{s}_{\mathrm{D}}\right)$ is a directed complete partial order. In the following, if confusion may arise, we refer to any structure built in this way as $D_{\mathrm{dcpo}}$. As observed in 2.1.4.(2) $\Sigma(D)$ is the collection of Scott opens on $D$. Given a per $A$ the intrinsic preorder, $\leq_{A}$, induces a preorder on $|A|, s_{A}$, defined as follows: $\forall d, e \in|A| . d \approx_{A} e \Leftrightarrow[d]_{A} s_{A}[e]_{A}$. Equivalently we have: $\forall \mathrm{d}, \mathrm{e} \in|\mathrm{A}|$. (ds $\mathrm{A} \Leftrightarrow \forall \phi \in|\mathrm{A} \rightarrow 1|$. $\phi \mathrm{d} \downarrow \Rightarrow \phi \mathrm{e} \downarrow)$.

\subsubsection{Definition (D-completeness)}

Given $a$, say, binary relation $R$ on $D_{\text {dcpo }}$, we say that $R$ is $D$-complete iff $X \subseteq R, X$ directed $\Rightarrow \cup_{D \times D} X \in R .15$

\subsubsection{Theorem $\left(c_{D}(\Sigma)\right.$ per ${ }_{\text {Ddcpo } 0} \varsigma_{>}(\Sigma)$ per $\left.{ }_{\text {Ddcpo }}\right)$}

The full subcategory of $D$-complete $(\Sigma)$ pers over $D_{\text {dcpo }}$ is reflective in $(\Sigma)$ per. Proof

One shows that if $A \in$ per and $B \in C_{D}$ per then $B A=B \underline{A}$ where $\underline{A}$ is the least $C_{D}$ per containing $A$. First one gives an inductive definition of $\underline{A}$ and then one can prove $B^{A}=$ $B \underline{A}$ by induction (see Amadio[89]). Finally observe that if $A$ is $D$-complete then $L_{\Sigma}(A)$ is $D-$ complete (see 2.3.1 for $\mathrm{L}_{\Sigma}$ definition).

\subsubsection{Remark}

We can exploit the continuity properties of the realizability structure in order to interpret a fixed point combinator on terms. Suppose $\left(D, s_{D}\right)$ has a least element denoted with $\perp_{D}$. As usual one can define a fixed point combinator on $D$ as: Fix $x_{D} \triangleq \lambda f . \cup_{n<\omega^{f}} n^{n}\left(\perp_{D}\right)$.

Say that $A$, per, is pointed iff $\perp_{D} A \perp_{D}$. Suppose that $A$ is a pointed, $D$-complete per. Define: $\operatorname{Fix}_{A} \stackrel{\Perp}{=}\left[\mathrm{Fix}_{D}\right]_{A} A_{\rightarrow A}$. It is easy to show (Amadio[89]) that Fix $A$ is a fixed point combinator on $\mathrm{A}$.

Actually if $A$ is separated then Fix $A$ returns the least fixed point w.r.t. the intrinsic order. The proof of this fact follows the standard argument adapted to the intrinsic order.

Also observe that given a per $A$ the preorder $s_{A}$ is $D$-complete. The proof is immediate from the characterization in 5.1.1. On the other hand we still lack a result stating some form of continuity of the realized maps w.r.t. the intrinsic order, a solution to this problem will be given in 5.3.

\subsection{Uniform $\mathrm{c}_{\mathrm{D}} \Sigma$ per $\left(\mathrm{c}_{\mathrm{D}} \mathrm{u} \Sigma\right.$ per)}

Suppose that $D_{\text {dcpo }}$ is built out of an inverse limit technique. The domain $D$ is then naturally stratified in levels. Given a per $A$ we can define its approximation at the $n$-th level $A_{1 n}$. It is then natural to consider a condition of uniformity that guarantees:

$\forall \mathbf{n} . \mathrm{A}_{\text {In }}=\mathrm{B}_{\text {In }} \Rightarrow \mathrm{A}=\mathrm{B}$.

To the best of our knowledge this condition first appears, with the name of "completeness under approximation" and quite different purposes, in Coppo[85].

\subsubsection{Assumption on the pca: $D_{\infty}$}

Let $D$ be a dcpo for which there is a sequence $\left\{p_{n} \mid p_{n}: D \rightarrow D\right\}_{n \in \omega}$ such that:

- $p_{n}$ is a projection: $p_{n}{ }^{\circ} p_{n}=p_{n} \leq i d_{D}$.

- $\left\{p_{n}\right\}_{n \in \omega}$ is a growing sequence with id as lub: $p_{0}=\lambda d . \perp_{D}, p_{n} \leq p_{n+1}, \cup_{n \in \omega}\left\{p_{n}\right\}=i d_{D}$. We also assume that the following dcpos can be embedded in the dcpo $\mathrm{D}$ by means of the 15 In the following to avoid confusion we denote with "lub" the lower upper bound in the quotient space and
with " $U$ " the lower upper bound in D. 
following embedding projection pairs that commute conveniently with the sequence of projections:
$\omega \quad\left(i_{\omega y} j_{\omega}\right) \quad p_{n+1} i_{\omega}=i_{\omega}$
(Numbers with flat order)
$\mathrm{D} \rightarrow \mathrm{D}$ :
$\left(i_{-,} j_{\perp}\right)$
$p_{n+1}\left(i_{-}(f)\right)=i_{\rightarrow}\left(p_{n} \circ f \circ p_{n}\right)$
(Partial Functions)
$\mathrm{D} \times \mathrm{D}$ :
$\left(i_{x}, j_{x}\right)$
$p_{n+1} i_{x}=i_{x} 0<p_{n}, p_{n}>$
(Product)

In practice such a $D$ can be exhibited by means of an inverse limit construction (Scott[72]), therefore we refer to a $D_{d c p o}$ satisfying all the previous conditions with $D_{\infty}$.

\subsubsection{Definition (uniformity)}

Given a binary relation $R$ on $D_{\infty}$, we say that $R$ is uniform iff $\mathrm{R} \neq \varnothing \wedge \mathrm{dRe} \Rightarrow \forall \mathrm{n} \in \omega . \mathrm{d}_{\mathrm{n}} \operatorname{Re}_{\mathrm{n}}$

\section{Convention}

We write: $D_{n} \equiv p_{n}(D), A_{\mid n} \equiv A \cap\left(D_{n} \times D_{n}\right)$. Note that $D_{n}$ is uniform and if $A$ is uniform then $A_{1 n}$ is uniform.

\subsubsection{Definition (metric on $c_{D}$ uper ${ }_{D_{\infty}}$ )}

Let $A, B \in C_{D}$ uper $_{D_{\infty}}$. Define $c(A, B) \triangleq \max \left\{n \mid A_{\mid n}=B_{\mid n}\right\}, d(A, B) \triangleq 2-c(A, B)$, where conventionally: $\max \omega \triangleq \infty$, and $2^{-\infty} \triangleq 0$.

\subsubsection{Facts (on $c_{D} u(\Sigma) p e r$, see Amadiof891)}

(1) $\left(c_{D}\right.$ uper $_{D}, d$ ) is a complete ultra-metric space. (2) The operators of exponentiation and product are contractive. (3) $C_{D}$ uper $_{D}$ is closed under arbitrary intersections. (4) The full subcategory of $\mathrm{C}_{\mathrm{D}}$ upers is cartesian closed.

\subsubsection{Remark (limits of the uniformity condition)}

The category $c_{D}$ uper lacks equalizers and closure under retraction. In particular for equalizers observe that a per composed by the equivalences classes $\{\{d\}$, $\{e\}\}$ where $d$ and $e$ are incomparable is not isomorphic to any cuper. In fact any cuper containing two incomparable equivalences classes would also include a least equivalence class. The problem of the lack of equalizers appears more serious than the one arising in categories of cpos and continuous maps.

Trying to fix this problem one may consider a notion of uniform map where $[\phi]: A \rightarrow B$ is uniform iff $\phi d_{n}=(\phi d)_{n}$. It is easy to check that equalizers of uniform maps are uniform. However uniform maps are strict and therefore do not behave well w.r.t. exponentiation. Another idea is needed.

\section{$5.3 \mathrm{c}_{\mathrm{D}} \mathrm{u} \Sigma$ per as domains}

Following Abadi\&Plotkin[90] we show that by requiring that the range of the projections $\mathrm{p}_{\mathrm{n}}$ is finite one can prove that if $A \in C_{D} u \Sigma$ per then the quotient space $D / A$ ordered with the intrinsic order is an sfp. Moreover all realized maps are continuous.

\subsubsection{Assumption on the pca: $D_{\text {sfp }}$}

We impose a further condition on $D_{\infty}$, namely we assume that for each $n \operatorname{rg}\left(p_{n}\right)$ is finite. Since this implies that $D_{\infty}$ is an sfp we refer to it with $D_{\text {sfp }}$. In this section (5.3) $D$ is an abbreviation for $D_{\text {sfp }}$.

After Abadi\&Plotkin[90], we characterize the induced preorder, $\approx_{A}(5.1 .1)$ as the least $D$ complete preorder containing $A \cup S_{D} \| A \mid$ (5.3.10). In other terms the intrinsic pre-order is as little as it could possibly be. Moreover $\leqslant_{A}$ turns out to be uniform (5.3.9). 


\subsubsection{Definition}

Let $<_{A} \triangleq T C(A \cup \leq D|| A \mid)$, where $T C \equiv$ Transitive Closure.

\subsubsection{Fact}

Proof

${ }_{\mathrm{A}}$ is a uniform preorder on $|\mathrm{A}|$.

Observe that $A \cup S_{D|| A \mid}$ is uniform as $A$ is uniform by assumption. Next remark that the operator of transitive closure, TC, preserves uniformity.

\subsubsection{Proposition}

Let $d \in D_{0}$, where $D_{0}$ is the collection of compact elements. Then $W(d) \triangleq\left\{e \in D|\exists e \in D \cap| A \mid . d<_{A} e^{\prime} \leq_{D} e\right\} \in \Sigma(D)$ that is a Scott open in D.

\section{Proof}

If $d \notin|A|$ then $W(d)=\varnothing . W(d)$ is clearly upward closed. Suppose $\left\{e_{i} \mid i \in I\right\}$ directed and such that $\cup\left\{e_{i} \mid i \in I\right\} \in W(d)$, that is: $d<_{A} e^{\prime} \leq_{D} \cup\left\{e_{i} \mid i \in I\right\}$. By the uniformity of $<_{A}$ and the compactness of $d: \exists n . d_{n}=d<_{A}\left(e^{\prime}\right)_{n} \leq_{D}\left(\cup\left(e_{i} \mid i \in I\right)\right)_{n}$. By the fact that $p_{n}$ has finite image: $\exists j . d<_{A}\left(e^{\prime}\right)_{n} \leq_{D}\left(e_{j}\right)_{n} \leq_{D} e_{j}$. Hence $e_{j} \in W(d)$.

\subsubsection{Note}

Given $d \in D_{0}$ define $U(d) \triangleq\left\{[e]_{A} \mid d<_{A}\right.$ e $\}$. Then $U(d) \in \Sigma(A)$ as $W(d) \cap|A|=|U(d)|$ (see 2.2.1 for def. $\Sigma(\mathrm{A})$ ).

\subsubsection{Corollary}

Given $d$ compact then $d<_{A} e$ iff $d \leqslant_{A} e$. Proof

In general $\leqslant_{A}$ contains $<_{A}$. Vice versa, by contradiction, suppose $d \approx_{A} e$ and $\neg\left(d<_{A} e\right)$. Build the Scott open $W(d) \in \Sigma(D)$ as in prop. 5.3.4 and the semi-computable subset $U(d)$ as in note 5.3.5. Then $[d]_{A} \in U(d)$ and $[e]_{A} \notin U(d)$. Contradiction.

\subsubsection{Definition}

Let $\prec_{\mathrm{AC}}$ be the least $\mathrm{D}$-complete preorder containing $<_{\mathrm{A}}$.

\subsubsection{Proposition}

\section{Proof}

${ }_{\mathrm{Ac}}$ coincides with $\approx_{\mathrm{A}}$.

By 5.1.4 $\approx_{A}$ is D-complete moreover it contains $<_{A}$ and therefore $<_{A c}$. Vice versa suppose $d \leqslant_{A} e$. Then $\forall n . d_{n} \leqslant_{D} d \leqslant_{A}$ e. Since $\leqslant_{A}$ contains $\leq_{D \mid l A l}$ we have $\forall n . d_{n} \leqslant_{A} e$. By 5.3.6 $\forall n . d_{n}{ }_{A} e$ and by completeness $d=U\left(d_{n} \mid n \in \omega\right)<A c e$.

\subsubsection{Proposition}

\section{Proof}

${ }_{A C}$ is uniform.

Give a transfinite inductive definition of the completion process and observe that uniformity is preserved at each step (see also Amadio[89]).

\subsubsection{Corollary}

The preorder, $\approx_{A}$, induced by the intrinsic preorder on $|\mathrm{A}|$ is the least D-complete preorder on $|A|$ containing $A \cup \leq D|| A \mid$.

Proof

Immediate from 5.3.8, 5.3.9. 
The interesting fall-out of the characterization of the intrinsic preorder is the following theorem that explicits the domain structure of the quotient space. The proof shows how to extract from a directed set $\left\{\left[d_{i}\right]_{A} \mid i \in I\right\}$ in $\left(D / A, \leq_{A}\right)$ a chain $\left\{e_{n} \mid n \in \omega\right\}$ in $D$ such that $e_{n} \in D_{n} \cap|A|$ and $\operatorname{lub}\left\{\left[d_{i}\right]_{A} \mid i \in I\right\}=\left[\cup\left\{e_{n} \mid n \in \omega\right]\right]_{A}$.

\subsubsection{Theorem (The quotient space is a cpo)}

Let $A$ be a $c_{D} u \Sigma$ per then $\left(D / A, \leq_{A}\right)$ is a cpo.

Proof (after Abadis Plotkin[90])

Clearly under the hypothesis $A \in \Sigma$ per $\left(D / A, s_{A}\right)$ is a partial order with least element $\left[\perp_{D}\right]_{A}$. Given a directed set, say $\left\{x_{i} \mid i \in I\right\} \triangleq\left\{\left[d_{i}\right]_{A} \mid i \in I\right\}$, we show that it has a lub in $\left(D / A, \leq_{A}\right)$. Given $\mathrm{J}^{\prime} \subseteq \mathrm{J} \subseteq \mathrm{I}$ we say that $\mathrm{J}^{\prime}$ is cofinal with $\mathrm{J}$ if $\forall i \in J . \exists j \in J^{\prime} \cdot\left(d_{i} \leqslant A \quad d_{j}\right)$.

Let $X_{n} \triangleq\left\{e \mid \forall i \in I . \exists j \in I .\left(d_{i} \leqslant A d_{j} \wedge e=\left(d_{j}\right)_{n}\right\}\right.$. In other words $e \in X_{n}$ if there is a subset $J$ of $I$, cofinal with $I$, and such that $\forall j \in J . e=\left(d_{j}\right)_{n}$.

a. $X_{n} \subseteq D_{n} \cap|A|$ is finite since $r g\left(p_{n}\right)$ is finite.

Assuming the directed set infinite (o.w. there is nothing to prove), again from the finiteness of $\mathrm{rg}\left(\mathrm{p}_{\mathrm{n}}\right)$, follows that each $X_{n}$ is non-empty as at least an element will be hit infinitely often.

b. $\forall \mathrm{e} \in \mathrm{X}_{\mathrm{n}} \cdot \exists \mathrm{e}^{\prime} \in \mathrm{X}_{\mathrm{n}+1} \cdot \mathrm{e} \leq_{\mathrm{D}} \mathrm{e}^{\prime}$.

Proceed by induction on $n$. If $n=0$ then $e=\perp$ and every $e^{\prime}$ will do. If $e \in X_{n}$ then there is a J, cofinal with $I$, such that $J \subseteq I$ and $\forall j \in J \cdot\left(d_{j}\right)_{n}=e$. Since $r g\left(p_{n}\right)$ is finite there is a J $\subseteq J$, cofinal with $J$, such that $\forall j \in J^{\prime} .\left(d_{j}\right)_{n+1}=e^{\prime}$, for some $e^{\prime}$. Observe for $j \in J^{\prime}: e=\left(d_{j}\right)_{n} \leq_{D}\left(d_{j}\right)_{n+1}=e^{\prime}$. Hence pick up e' in $X_{n+1}$. The result of this process is that we can choose a sequence $\left\{e_{n} \mid n \in \omega\right\}$ such that $e_{n} \in X_{n}$ and $e_{n} \leq_{D} e_{n+1}$.

By D-completeness we have that $\cup\left\{e_{n} \mid n \in \omega\right\} \in|A|$. The claim is:

$\exists \operatorname{lub}\left\{\left[\mathrm{d}_{\mathrm{i}}\right]_{\mathrm{A}} \mid \mathrm{i} \in \mathrm{I}\right]=\left[\cup\left(\mathrm{e}_{\mathrm{n}} \mid \mathrm{n} \in \omega\right)\right]_{\mathrm{A}}$.

In the first place let us show: $\forall i \in I . d_{i} \leqslant_{A} \cup\left\{e_{n} \mid n \in \omega\right\}$. By completeness and uniformity it is enough to show that: $\forall \mathrm{i} \in I . \forall \mathrm{m} \in \omega .\left(\mathrm{d}_{\mathrm{i}}\right)_{\mathrm{m}} \approx_{A} \cup\left\{\mathrm{e}_{\mathrm{n}} \mid \mathrm{n} \in \omega\right\}$. Now:

$\forall i \in I . \forall m \in \omega . \exists j \in I .\left(d_{i} s_{A} d_{j} \wedge\left(d_{j}\right)_{m}=e_{m} \wedge e_{m} \leq_{D} \cup\left\{e_{n} \mid n \in \omega\right]\right)$,

hence by the characterization of $\leqslant_{A}$ we can conclude: $\left(d_{i}\right)_{m} \leqslant_{A}\left(d_{j}\right)_{m} \leqslant A \cup\left\{e_{n} \mid n \in \omega\right\}$ that gives the desired assertion.

Finally we prove that: $\forall n \in \omega . \exists i \in I . e_{n} \leqslant_{A} d_{i}$. For some $i_{s} e_{n}=\left(d_{i}\right)_{n} \leq_{D} d_{i}$ that implies $e_{n} \approx_{A} d_{i}$. Hence given $[d]_{A}$ majorant for $A$ it is immediate to show: $\cup\left\{e_{n} \mid n \in \omega\right\} \leqslant A d$.

\subsubsection{Corollary (realized maps are continuous)}

Let $A, B c_{D} u \Sigma$ pers and $f: A \rightarrow B$ a morphism in the category. Then $f$ is a continuous map w.r.t. the domain structure of the quotient spaces.

Proof

Take $f: A \rightarrow B,\left\{x_{i} \mid i \in I\right\} \triangleq\left\{\left[d_{i}\right]_{A} \mid i \in I\right\}$ directed in $D / A$. The existence of lub $\left\{f\left(x_{i}\right) \mid i \in I\right\}$ is guaranteed by 5.3.11 and the monotonicity of $f$. We just have to show that:

$f\left(l u b\left\{x_{i} \mid i \in I\right\}\right) \leq_{A}$ lub $\left\{f\left(x_{i}\right) \mid i \in I\right\}$.

Suppose $\phi$ realizes $f$ and consider the chain $\left\{e_{n} \mid n \in \omega\right\}$ built in 5.3.11. Then we have: $\phi\left(\cup\left\{e_{n} \mid n \in \omega\right\}\right)=\cup\left\{\phi e_{n} \mid n \in \omega\right\}$ and we observe that: $\forall n \in \omega . \exists i \in I . \phi e_{n} \approx B \phi d_{i}$, hence we can conclude $\left[\cup\left\{\phi e_{n} \mid n \in \omega\right)\right]_{B} \approx_{B} \operatorname{lub}\left\{f\left(x_{i}\right) \mid i \in I\right\}$.

\subsubsection{Corollary (the quotient space is an $s f p$ )}

Let $A$ be a $c_{D} u \Sigma$ per then $\left(D / A, \leq_{A}\right)$ is an sfp. 


\section{Proof}

Consider the sequence $\left\{\pi_{n} \mid n \in \omega\right\}$ where: $\pi_{n}: A \rightarrow A$ is the map realized by $p_{n}$ as specified in 5.2.1 and 5.3.1. All the checks follow immediately by the properties of $\left\{p_{n} / n \in \omega\right\}$.

\section{Conclusion}

Much work remains to be done. In the first place there is a strong need for the development and refinement of the theory. For example the categories of pers over $(\omega, \cdot)$ built in sections 3 and 4 do not seem to satisfy enough algebraicity and effectivity properties (see 3.2.6.(2)). More research is needed in this direction. Taking another approach it has been shown in section 5 that it is possible to define a category of pers that enjoys many properties of standard categories of domains. This result is achieved by imposing a condition of uniformity whose categorical properties are not completely satisfying. This should stimulate further investigation.

On the other hand there is a wide spectrum of possible applications. So far the main one has been in the area of typed functional programming languages. cuspers can be considered as a model for a functional, typed language, based on Girard's system $F$ extended with a fixed-point combinator on types and subtyping.

Adequacy results with respect to some standard "operational semantics" can be obtained rather easily. The basic property is roughly the following: let $M$ be a term of type $\alpha$ and let er(M) be the type free lambda term underlying $M$ (for simplicity assume there are no free

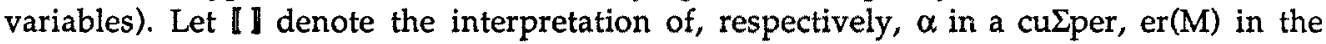
realizability structure $\mathrm{D}$, and $\mathrm{M}$ in an equivalence class of $\llbracket \alpha \rrbracket$. It can be proved that:

$\llbracket M \rrbracket=[\llbracket e r(M) \rrbracket]_{[\alpha \rrbracket}$. From this one can apply classical results relating the typed calculus to the type-free calculus as well as the type-free calculus to the realizability structure D (see, e.g., Amadio[89]).

More original insights can be obtained in the modelling of subtyping. See, for example, the proposal for the "Quest" programming language in Cardelli\&Longo[90]. Amadio\&Cardelli[90] illustrates the role of cupers in modelling the subtyping of recursive types. Another potential field of application is the interpretation of extensions of calculi of constructions with recursive definitions. The structures introduced are then relevant in proving the consistency of such extensions and in optimizing the process of programs' extraction. See Paulin-Mohring[89] for a proposal related to Coquand-Huet calculus of constructions. Finally let us mention that Streicher[89] exploits extensional pers to study consistency and interrelations of various category-theoretic axiomatizations of calculi of dependent types.

\section{Acknoweldgments}

Pierre-Louis Curien and Giuseppe Longo, made comments on a preliminary version of this paper. Thanks to Pino Rosolini and Thomas Streicher for discussions.

\section{References ${ }^{16}$}

Abadi M., Plotkin G. [1990] "A per model of polymorphism and recursive types", 5th IEEE-LICS, Philadelphia. Amadio R. [1989] "Recursion over realizability structures", TR 1/89 Dipartimento di Informatica, Università di Pisa, (to appear on Info.\&Comp.).

Amadio R., Cardelli L. [1990] "Subtyping Recursive Types", DEC-SRC TR \#62, ext. abs. to appear in Proc. ACMPOPL91.

Bruce K, Longo G. [1990] "A modest model of records, inheritance and bounded quantification", Info\&Comp., 87, 1-2, (196-240), Preliminary version in Proc. of IEEE-LICS 88.

Carboni A., Freyd P., Scedrov A. [1987] "A categorical approach to realizability and polymorphic types" 3rd

\footnotetext{
16 While this paper goes to the press new results are announced by many people including Hyland, Phoa, Rosolini, and Taylor. Unfortunately it is impossible for us to report on them as well as to give exact references.
} 
ACM Symp. on Math. Found of Language semantics, New Orleans.

Cardelli L., Longo G. [1990] "A semantic basis for Quest", in Proc LISP\&FP, Nice.

Coppo M. [1985] " A completeness theorem for recursively defined types", in Proc. 12th ICALP, Nafplion, Greece 1985, SLNCS 194.

Curien P-L., Obtulowicz A. [1988] "Partiality, Cartesian Closedness and Toposes", Information \& Computation, 80, (50-95).

Droste M., Göbel R. [1990] "Universal domains in the theory of denotational semantics of programming languages", IEEE-LICS 90, Philadelphia.

Freyd P., Mulry P., Rosolini G., Scott D. [1990] "Extensional Pers", 5th IEEE-LICS, Philadelphia.

Gunter C., Jung A. [1990] "Coherence and Consistency in Domains", J.P.Apl.Alg., 63, (49-66),

Hyland M. [1982] "The effective Topos," in The Brouwer Symposium, (Troelstra, Van Dalen eds.), NorthHolland.

Hyland M. [1988] "A small complete category", APAL, 40, 2, (135-165).

Longo G., Moggi E. [1984] "Cartesian closed categories of enumerations for effective type-structures", in Symp. on Semantics of Data Types, Kahn\&al. (eds.), SLNCS 173.

Longo G., Moggi E. [1988] "Constructive Natural Deduction and its Modest Interpretation", CMU Report CS-88131, Lecture delivered at the workshop "The semantics of natural and programming languages" Stanford, March 1987.

Mac Lane S. [1971] "Categories for the working mathematician", Springer-Verlag, New York.

McCarthy D.[1984] "Realizability and Recursive mathematics" Ph. D. Thesis, Merton College, Oxford.

Moggi E. [1988] "Partial morphisms in categories of effective objects", Info.\&Comp., 76, (250-277).

Mulry P. [1981] "Generalized Banach-Mazur functionals in the topos of recursive sets", J. Pure Apl. Algebra, 26, 1, (71-83).

Myhill J., Shepherdson J. [1955] "Effective operations on partial recursive functions", Zeit. für Math. Logik und Grund. der Math., 1, (310-317).

Paulin-Mohring C. [1989] "Extraction de programmes dans le calcul des contructions", thèse, Paris VII.

Phoa W. [1990] "Effective domains and intrinsic structure", 5th IEEE-LICS, Philadelphia.

Plotkin G. [1980/4] "Domains", lecture notes, C.S. Dept., Edinburgh.

Robinson E., Rosolini P. [1988] "Categories of partial maps", Info\&Co., 79, (95-130).

Rosolini G. [1986] "Continuity and effectiveness in Topoi" PhD Thesis, Oxford University.

Scott D. [1976] "Data types as lattices," SIAM Journal of Computing, 5 (pp. 522-587).

Smyth M. [1977] "Effectively Given Domains", Theoret. Comput. Sci. 5, (255-272).

Smyth M., Plotkin G. [1982] "The category-theoretic solution of recursive domain equations" SIAM Journal of Computing 11, (pp.761-783).

Soare R. [1987] "Recursively enumerable sets and degrees", Springer-Verlag.

Streicher T. [1990] "Independence results for impredicative calculi of dependent types", preprint.

Troelstra A., van Dalen D. [1988] "Constructivism in Mathematics", 2 vols. , North-Holland.

Wand M. [1979] "Fixed-point Constructions in Order-enriched Categories", Theoret. Comp. Sci. (13-30). 